\title{
Generalized semiclassical-phase-index formulas via sequential stationary phase
}

\author{
G. Campolieti and Paul Brumer \\ Chemical Physics Theory Group, Department of Chemistry, University of Toronto, Toronto, Canada M5S 1A1
}

(Received 19 May 1994; revised manuscript received 3 October 1995)

\begin{abstract}
Sequential stationary phase, i.e., the replacement of a multidimensional stationary phase evaluation by an ordered set of lower-dimensional stationary phase integrations, is applied to uniformly asymptotic path integral forms for the semiclassical propagator. The results are useful formulas for computing trajectory indices (generalized Maslov indices) in any quantum representation for general types of time-dependent Hamiltonians. Index connecting relations that relate the indices for the different representations are also obtained. We also demonstrate a general canonical structure for the semiclassical phase indices that arises naturally through the application of sequential stationary phase.
\end{abstract}

PACS number(s): 03.65.Sq

\section{INTRODUCTION}

Semiclassical mechanics provides useful expressions for quantum matrix elements and related quantities in the $\hbar \rightarrow 0$ limit. Of particular interest are matrix elements of the propagator in various representations (i.e., coordinate space, momentum space or mixed coordinate-momentum space representations). These are typically of the form of an amplitude, which depends upon the classical stability matrix and an exponent, which depends upon the classical action [1]. In addition, the exponents contain important phase terms, generally referred to as Maslov indices, which arise from the trajectory crossing various caustics. This semiclassical structure was demonstrated in early stationary-phase path-integral treatments of the coordinate representation of the propagator for kinetic-plus-potential-type Hamiltonians [2,3]. These important indices also arise within a much more general context within semiclassical formulas.

Modern treatments of the theory of Maslov indices tend to be complicated, relying upon sophisticated mathematical language [4]. In a recent paper we provided a straightforward derivation of the Maslov indices in various representations [5]. Specifically, by concatenating uniform short-time propagator formulas for the coordinate and/or momentum representations and applying stationary-phase methods on the resulting path-integral form we obtained general Maslov index formula for any type of caustic and for quite general Hamiltonians. Our treatment [5] suggested the possibility of both developing an underlying consistent canonical structure of semiclassical matrix elements and obtaining the purely classical canonical properties of the general Maslov indices, with the phase indices embedded in the semiclassical formulas in a consistent manner. This paper provides such a development for the propagator, starting from an asymptotic path-integral formulation for more general types of time-dependent Hamiltonians and making extensive use of a single tool: sequential stationary phase. By sequential stationary phase, abbreviated SSP, we mean the reliable stationary-phase evaluation of multidimensional integrals via a set of stationaryphase evaluations on integrals of lower dimension.

The formulation developed in this paper is essentially as rigorous as that which relates asymptotic properties of wave functions to Lagrangian manifolds in phase space [4]. Al- though intimately connected to it, our work does not rely on the formal language of differentiable manifolds, which, although of geometrical appeal, requires sophisticated mathematical tools. The key point is that all that we require is the implicit function theorem and basic tools in classical mechanics. Moreover, our treatment emphasizes simple and computationally useful trajectory aspects of the theory.

The paper is organized as follows. In Sec. II we discuss some formal aspects of sequential stationary phase that are necessary for its validity. Section III contains the pathintegral development of the semiclassical propagator matrix elements and of the trajectory index formulas for various representations. The general formulas for the indices is given in Sec. III A, where their inherent canonical structure is displayed. Moreover, we explore important index properties, such as index contributions to a trajectory from the joining of trajectory segments. Useful "connecting relationships" for relating the various indices are given in Sec. III B. Section IV contains a summary. Three appendixes provide supporting material: Appendix A discusses conditions for the validity of sequential stationary phase, Appendix B contains useful equations on classical generators, and Appendix C contains useful expressions relating to matrix signatures and the symplectic properties of classical mechanics.

\section{SEQUENTIAL STATIONARY PHASE}

Consider a multidimensional integral of the form

$$
I(\hbar ; \boldsymbol{\alpha})=\int d \mathbf{x} \int d \mathbf{y} g(\mathbf{x}, \mathbf{y} ; \boldsymbol{\alpha}) \exp \left[\frac{i}{\hbar} \varphi(\mathbf{x}, \mathbf{y} ; \boldsymbol{\alpha})\right]
$$

where the integration is over a bounded or unbounded domain in $d=m+n$ dimensions with $(\mathbf{x}, \mathbf{y}) \in\left(\mathbb{R}^{m}, \mathbb{R}^{n}\right)$, for integer $m, n$. The functions $g$ and $\varphi$ are real valued within the domain of integration and sufficiently continuously differentiable functions in $\mathbf{x}, \mathbf{y}$ so that an asymptotic expansion follows via stationary phase. For generality, we have included in Eq. (1) a dependence on a space of parameters $\boldsymbol{\alpha}$, which is not explicitly carried below but will be of use later.

Semiclassical mechanics relies upon stationary-phase approximations to integrals such as those in Eq. (1). The critical points of this integral are assumed to be nondegenerate, 
isolated real interior stationary-phase points of $\varphi$. These stationary-phase points are a finite number $s$ of points $(\mathbf{x}, \mathbf{y})=\left(\mathbf{x}_{*}^{(k)}, \mathbf{y}_{*}^{(k)}\right), k=1, \ldots, s$, that solve the system

$$
\partial_{\mathbf{x}} \varphi=\mathbf{0}, \partial_{\mathbf{y}} \varphi=\mathbf{0}
$$

uniquely with $\operatorname{det} \partial_{\left(\mathbf{x}_{*}, \mathbf{y}_{*}\right)}^{2(k)} \varphi \neq 0$. [Throughout this paper the following compact notation is used: $\partial_{\mathbf{v}} \mathbf{u}$ and $\partial_{\mathbf{v}} \partial_{\mathbf{u}} f$ denote matrices with elements in the $i$ th row and $j$ th column given by $\partial u^{i} / \partial v^{j}$ and $\partial^{2} f / \partial u^{i} \partial v^{j}$, respectively, for any function $f(\mathbf{u}, \mathbf{v})$ and vectors $\mathbf{u}=\left(u^{1}, \ldots, u^{M}\right), \mathbf{v}=\left(v^{1}, \ldots, v^{N}\right)$ with $(\mathbf{u}, \mathbf{v}) \equiv\left(u^{1}, \ldots, u^{M}, v^{1}, \ldots, v^{N}\right)$. Hessian matrices are given by $\left(\partial_{\mathbf{w}}^{2} \varphi\right)_{i j}=\partial^{2} \varphi / \partial w^{j} \partial w^{i}$ for any vector $\mathbf{w}$. Vectors multiplying to the left (right) of a matrix are taken as row (column) vectors. The notation $\partial_{\left(\mathbf{x}_{*}, \mathbf{y}_{*}\right)}$, etc., denotes $\partial_{\mathbf{x}} \partial_{\mathbf{y}}$ evaluated at $\mathbf{x}=\mathbf{x}_{*}, \mathbf{y}=\mathbf{y}_{*}$.] The standard stationary-phase method [6,7] gives the $\hbar \rightarrow 0$ leading asymptotic term

$$
\begin{aligned}
I(\hbar) \sim I^{\mathrm{SP}}(\hbar)= & (2 \pi \hbar)^{d / 2}\left|\operatorname{det} \partial_{\left(\mathbf{x}_{*}, \mathbf{y}_{*}\right)}^{2} \varphi\right|^{-1 / 2} g\left(\mathbf{x}_{*}, \mathbf{y}_{*}\right) \\
& \times \exp \left[\frac{i}{\hbar} \varphi\left(\mathbf{x}_{*}, \mathbf{y}_{*}\right)+i \frac{\pi}{4} \operatorname{sig} \partial_{\left(\mathbf{x}_{*}, \mathbf{y}_{*}\right)}^{2} \varphi\right] .
\end{aligned}
$$

Here and below there is an implied sum over all stationaryphase points, which are simply denoted by $\left(\mathbf{x}_{*}, \mathbf{y}_{*}\right)$. Here $\operatorname{sig} \mathbf{M}=(d-2 \mu)$ is the signature of $\mathbf{M}$ defined as the number of positive $d-\mu$ minus the number of negative $\mu$ eigenvalues of the real-symmetric matrix $\mathbf{M}$. We refer to $I^{\mathrm{SP}}(\hbar)$ as the complete stationary-phase approximation to $I(\hbar)$.

In semiclassical mechanics it is common to apply the stationary-phase approximation sequentially, i.e., to divide up the integral in Eq. (1) into a stationary-phase approximation for the $\mathbf{y}$ integral first, followed by a stationary-phase evaluation of the remaining $\mathbf{x}$ space integral. Conditions under which this procedure, which we term "sequential stationary-phase," yields the full stationary-phase result [Eq. (3)] are summarized below. (A full discussion of this approach is provided in Appendix A.) Moreover, we note some simple differential properties, which will be exploited throughout the entire phase index theory that follows.

First, let the continuously differentiable function $\tilde{\mathbf{y}}$ mapping $\mathbf{x} \in \mathbb{R}^{m}$ into $\widetilde{\mathbf{y}}(\mathbf{x}) \in \mathbb{R}^{n}$ be given by

$$
\partial_{\mathbf{y}} \varphi(\mathbf{x}, \widetilde{\mathbf{y}}(\mathbf{x}))=\mathbf{0} \text { for any } \mathbf{x} .
$$

Assume the sub-Hessian condition

$$
\left.\operatorname{det} \partial_{\mathbf{y}}^{2} \varphi(\mathbf{x}, \mathbf{y})\right|_{\mathbf{y}=\widetilde{\mathbf{y}}(\mathbf{x})} \neq 0 \text { for any } \mathbf{x}
$$

and that the function $\widetilde{\varphi}(\mathbf{x}) \equiv \varphi(\mathbf{x}, \widetilde{\mathbf{y}}(\mathbf{x}))$ has a critical point $\mathbf{x}^{*}$,

$$
\partial_{\mathbf{x}} \widetilde{\varphi}\left(\mathbf{x}^{*}\right)=\mathbf{0}
$$

then three properties hold [8]: (i) $\left(\mathbf{x}_{*}, \mathbf{y}_{*}\right)=\left(\mathbf{x}^{*}, \widetilde{\mathbf{y}}\left(\mathbf{x}^{*}\right)\right)$ is a critical point of $\varphi$ [i.e., satisfying Eqs. (2)], (ii) the following product formula for the Hessian determinants holds:

$$
\operatorname{det} \partial_{(\mathbf{x}, \mathbf{y})}^{2} \varphi(\mathbf{x}, \widetilde{\mathbf{y}}(\mathbf{x}))=\operatorname{det} \partial_{\mathbf{y}}^{2} \varphi(\mathbf{x}, \widetilde{\mathbf{y}}(\mathbf{x})) \operatorname{det} \partial_{\mathbf{x}}^{2} \widetilde{\varphi}(\mathbf{x}),
$$

(iii) as does the signature property

$$
\operatorname{sig} \partial_{(\mathbf{x}, \mathbf{y})}^{2} \varphi(\mathbf{x}, \widetilde{\mathbf{y}}(\mathbf{x}))=\operatorname{sig} \partial_{\mathbf{y}}^{2} \varphi(\mathbf{x}, \widetilde{\mathbf{y}}(\mathbf{x}))+\operatorname{sig} \partial_{\mathbf{x}}^{2} \widetilde{\varphi}(\mathbf{x}) .
$$

In particular, properties (ii) and (iii) hold for $\mathbf{x}=\mathbf{x}_{*}$. Note, here and below, the particular compact notation, e.g., $\partial_{\mathbf{x}} \partial_{\mathbf{y}} \varphi(\mathbf{x}, \widetilde{\mathbf{y}}(\mathbf{x}))$, which implies that one takes both $\mathbf{x}$ and $\mathbf{y}$ derivatives before substituting $\mathbf{y}=\widetilde{\mathbf{y}}(\mathbf{x})$. Appendix A contains a proof of properties (i)-(iii) given Eqs. (4)-(6). Of importance is the implications of these properties to the utility of sequential stationary-phase operations. In particular, we have the important following corollary: if Eqs. (4)-(6) hold, then the stationary-phase approximation in Eq. (3) applied successively on $\mathbf{y}$ and then $\mathbf{x}$ subspaces to the integral in Eq. (1) gives the same result as the complete stationaryphase approximation [Eq. (3)]. This corollary follows by applying the stationary-phase on the $n$-dimensional $\mathbf{y}$ integral first:

$$
\begin{aligned}
I_{1}^{\mathrm{SP}}(\hbar)= & (2 \pi \hbar)^{n / 2} \int d \mathbf{x}\left|\operatorname{det} \partial_{\mathbf{y}}^{2} \varphi(\mathbf{x}, \widetilde{\mathbf{y}}(\mathbf{x}))\right|^{-1 / 2} g(\mathbf{x}, \widetilde{\mathbf{y}}(\mathbf{x})) \\
& \times \exp \left[\frac{i}{\hbar} \widetilde{\varphi}(\mathbf{x})+i \frac{\pi}{4} \operatorname{sig} \partial_{\mathbf{y}}^{2} \varphi(\mathbf{x}, \widetilde{\mathbf{y}}(\mathbf{x}))\right] .
\end{aligned}
$$

From assumptions in Eqs. (5) and (6), this integrand's amplitude is continuous, with $\widetilde{\varphi}$ continuously differentiable, and we can again apply the stationary-phase method to this integral. According to property (i) this picks up all of the complete stationary-phase points of $\varphi$, giving the final sequential result

$$
\begin{aligned}
I_{1,2}^{\mathrm{SP}}(\hbar)= & (2 \pi \hbar)^{d / 2} \frac{g\left(\mathbf{x}_{*}, \mathbf{y}_{*}\right)}{\left|\operatorname{det} \partial_{\mathbf{y}}^{2} \varphi\left(\mathbf{x}_{*}, \mathbf{y}_{*}\right) \operatorname{det} \partial_{\mathbf{x}_{*}}^{2} \widetilde{\varphi}\right|^{1 / 2}} \\
& \times \exp \left[\frac{i}{\hbar} \varphi\left(\mathbf{x}_{*}, \mathbf{y}_{*}\right)+i \frac{\pi}{4}\left[\operatorname{sig} \partial_{\mathbf{y}}^{2} \varphi\left(\mathbf{x}_{*}, \mathbf{y}_{*}\right)\right.\right. \\
& \left.+\operatorname{sig} \partial_{\mathbf{x}_{*}}^{2} \widetilde{\varphi}\right] .
\end{aligned}
$$

Properties (ii) and (iii) then finally prove the above corollary, namely, that $I_{1,2}^{\mathrm{SP}}=I^{\mathrm{SP}}$.

This result makes the usual assumptions of stationaryphase integration. The more subtle requirement to note is the sub-Hessian condition in Eq. (5). For general integrands this condition may be violated, resulting in a sequential stationary-phase result that differs from the full multidimensional stationary-phase result. Generally, however, and most importantly for the semiclassical theory below, the possible violation of the sub-Hessian conditions can only occur at special or particular subset values of measure zero in $\boldsymbol{\alpha}$, where the inverse of the sub-Hessian $\partial_{\mathbf{y}}^{2} \varphi\left(\mathbf{x}_{*}, \mathbf{y}_{*}\right)$ simply does not exist. A key point centers about making sure one obtains all the real stationary-phase points of the complete phase $\varphi$ when using a sequential procedure. The above result ensures us that this is the case if the sub-Hessian condition in Eq. (5) is satisfied for the global domain of the $\mathbf{x}$ integration.

A subtle and most useful point now follows: If the subHessian condition is satisfied locally about all $\left(\mathbf{x}_{*}, \mathbf{y}_{*}\right)$ [i.e., $\operatorname{det} \partial_{\mathbf{y}}^{2} \varphi(\mathbf{x}, \mathbf{y}) \neq 0$ at $\mathbf{x}=\mathbf{x}_{*}, \mathbf{y}=\mathbf{y}_{*}$, but not necessarily globally in all $\mathbf{x}$, then properties (i), (ii), and (iii) are also locally valid. Therefore, one is solving for all the complete 
stationary-phase points via Eq. (4) followed by use of Eq. (6); this is equivalent to using a priori knowledge of all the $\left(\mathbf{x}_{*}, \mathbf{y}_{*}\right)$ by solving Eqs. (2) directly. Putting this into Eq. (10) and using properties (ii) and (iii) gives $I_{1,2}^{\mathrm{SP}}=I^{\mathrm{SP}}$. Hence, as long as the signature terms in $I_{1,2}^{\mathrm{SP}}$, which are computed only at critical points, are well defined (i.e., the Hessian matrices involved there are nonsingular), then $I_{1,2}^{\mathrm{SP}}=I^{\mathrm{SP}}$.

Note further that repeated application of the argument above evidently shows that the above SSP process applies to an arbitrary sequence of multiple integrals. In fact, it is precisely the repeated application of properties (ii) and (iii) that we employ below within SSP operations to obtain a consistent semiclassical structure for unitary matrix element transformations and also to obtain general index formulas that connect the generalized semiclassical phase indices.

\section{SEMICLASSICAL PROPAGATOR MATRIX ELEMENTS AND THE TRAJECTORY INDEX FORMULAS}

We now show that by starting with an asymptotic pathintegral form in any chosen representation, then repeated sequential stationary-phase operations give rise to a closed canonical structure for the semiclassical propagator formulas in any representation, with the Maslov indices clearly displayed. The key is that canonical transformations involve a special class of integral transforms for which the stationaryphase conditions are just classical generating function relations. In particular, it is also shown here that the sequential stationary-phase process is a very general and simple method for generating all the purely classical properties of trajectoryphase indices and formulas that relate the Maslov index for the semiclassical formula in any one representation to that in any other. We explicitly prove and present the most useful properties of the indices, including "connecting relationships" that relate the various indices for the coordinate, momentum, and mixed representations. A canonical structure of the trajectory indices is also exposed. An alternate pathintegral framework is invoked and all index formulas are obtained solely on the basis of the SSP property, combined with classical generator identities.

\section{A. Semiclassical propagators and index formulas via SSP on path integration}

Consider any $N$ degree of freedom system with quantum Hamiltonian $\hat{H}=H(\hat{\mathbf{q}}, \hat{\mathbf{p}}, t)$ with canonical operators $\hat{\mathbf{q}}, \hat{\mathbf{p}}$. The operator $\hat{H}$ and its corresponding classical Hamiltonian in canonical variables $\mathbf{q}, \mathbf{p}$ is allowed to take on general forms, including kinetic-plus-potential Hamiltonians, fielddependent interactions with $\partial_{\mathbf{p}}^{2} H=\mathbf{m}^{-1}$ (inverse mass tensor), and more general forms in which $\partial_{\mathbf{p}}^{2} H$ is not necessarily constant. The canonical quantum operators $\hat{\mathbf{q}}$ and $\hat{\mathbf{p}}$ act on coordinate and momentum eigenstates $\hat{\mathbf{q}}|\mathbf{q}\rangle=\mathbf{q}|\mathbf{q}\rangle$ and $\hat{\mathbf{p}}|\mathbf{p}\rangle=\mathbf{p}|\mathbf{p}\rangle$, with $\mathbf{q}, \mathbf{p}$ values assumed on the infinite domain and normalization chosen here as $\langle\mathbf{q} \mid \mathbf{p}\rangle$ $=(2 \pi i \hbar)^{-N / 2} e^{i \mathbf{q} \cdot \mathbf{p} / \hbar}$. (N.B. $i \equiv e^{i \pi / 2}$ is the chosen branch throughout.) We assume a simply connected phase space throughout this work.

Our starting point lies in the use of general infinitesimaltime asymptotic formulas, which are concatenated to produce path-integral expressions for the propagator matrix el- ements in any representation, for global time. We need only use one such short-time formula, which for the initial momentum to final coordinate matrix element of the propagator $\hat{U}\left(t_{j+1}, t_{j}\right)$, is [5]

$$
\begin{aligned}
& \left\langle\mathbf{q}_{j+1}\left|\hat{U}\left(t_{j+1}, t_{j}\right)\right| \mathbf{p}_{j}\right\rangle \\
& \sim(2 \pi i \hbar)^{-N / 2}\left|\operatorname{det} \partial_{\widetilde{\mathbf{q}}_{j}} \mathbf{q}\left(\widetilde{\mathbf{q}}_{j}, \mathbf{p}_{j} ; t_{j+1}, t_{j}\right)\right|^{-1 / 2} \\
& \quad \times \exp \left\{\frac{i}{\hbar}\left[\phi\left(\mathbf{q}_{j+1}, \widetilde{\mathbf{q}}_{j} ; t_{j+1}, t_{j}\right)+\widetilde{\mathbf{q}}_{j} \cdot \mathbf{p}_{j}\right]\right\} \\
& \sim(2 \pi i \hbar)^{-N / 2} \exp \left\{\frac{i}{\hbar}\left[\phi\left(\mathbf{q}_{j+1}, \widetilde{\mathbf{q}}_{j} ; t_{j+1}, t_{j}\right)+\widetilde{\mathbf{q}}_{j} \cdot \mathbf{p}_{j}\right]\right\} .
\end{aligned}
$$

Here this is written in a form that will give rise to general path-integral forms convenient for what follows. Equation (11) involves a single classical root trajectory [9], which begins at time $t_{j}$ with momentum $\mathbf{p}_{j}$ and ends at time $t_{j+1}$ with coordinate $\mathbf{q}_{j+1}$ after having evolved over an infinitesimaltime interval $\left(t_{j+1}-t_{j}\right) \rightarrow 0$. The initial coordinate $\mathbf{q}_{j}=\widetilde{\mathbf{q}}_{j}\left(\mathbf{q}_{j+1}, \mathbf{p}_{j} ; t_{j+1}, t_{j}\right)$ is considered to be a function of $\mathbf{q}_{j+1}, \mathbf{p}_{j}, t_{j+1}$, and $t_{j}$ and is given implicitly by the root trajectory equation $\mathbf{q}\left(\widetilde{\mathbf{q}}_{j}, \mathbf{p}_{j} ; t_{j+1}, t_{j}\right)=\mathbf{q}_{j+1}$ or by one of the generator relations given in Appendix B,

$$
\mathbf{p}_{j}=-\partial_{\widetilde{\mathbf{q}}_{j}} \phi\left(\mathbf{q}_{j+1}, \widetilde{\mathbf{q}}_{j} ; t_{j+1}, t_{j}\right) .
$$

The classical action $\phi$ is the generator of classical motion expressed as a function of initial and final coordinates as defined by Eq. (B1) in Appendix B. The tilde linking the leftand right-hand sides of Eq. (11) indicates that the short-time form is asymptotic in $\hbar \rightarrow 0$, uniformly in $t_{j+1}-t_{j} \rightarrow 0$. This can be shown for very general Hamiltonians by putting Eq. (11) into the quantum evolution equation and using the usual time-dependent WKB treatment [4]. Although this asymptotic property is all that is needed as a building block in what follows, we note that for most Hamiltonians Eq. (11) also represents, for given $\hbar$, a uniformly asymptotic result in the limit $t_{j+1}-t_{j} \rightarrow 0$ and approaches the exact plane-wave result in this limit wherein $\hat{U}\left(t_{j+1}, t_{j}\right)$ becomes the identity operator.

Since this paper also deals with the general canonical structure of the semiclassical propagator matrix elements, we also introduce the following form, equivalent to Eq. (11). Specifically, the classical generator defined by the Legendre transform [i.e., Eq. (B4)] $\widetilde{\phi}=\widetilde{\phi}\left(\mathbf{q}_{j+1}, \mathbf{p}_{j} ; t_{j+1}, t_{j}\right)$ $=\phi\left(\mathbf{q}_{j+1}, \widetilde{\mathbf{q}}_{j} ; t_{j+1}, t_{j}\right)+\widetilde{\mathbf{q}}_{j} \cdot \mathbf{p}_{j}$ expresses the phase directly as function of the given initial momentum and final coordinate. The analogous generator relations are given by Eqs. (B5) and using the properties in Eqs. (B6), which follow, shows that the short-time propagator [Eq. (11)] has the alternate form

$$
\begin{aligned}
\left\langle\mathbf{q}_{j+1}\left|\hat{U}\left(t_{j+1}, t_{j}\right)\right| \mathbf{p}_{j}\right\rangle \sim & (2 \pi i \hbar)^{-N / 2}\left|\operatorname{det} \partial_{\mathbf{q}_{j+1}} \partial_{\mathbf{p}_{j}} \widetilde{\phi}\right|^{1 / 2} \\
& \times \exp \left[\frac{i}{\hbar} \widetilde{\phi}\left(\mathbf{q}_{j+1}, \mathbf{p}_{j} ; t_{j+1}, t_{j}\right)\right] .
\end{aligned}
$$


The canonical nature of the short-time matrix element is therefore manifest solely in terms of the appropriate generator, namely, $\widetilde{\phi}$. This aspect of the semiclassical formulas is well known [10]. Although short-time expressions for other representations also follow, they will not be explicitly needed throughout the treatment given in this paper.

A path-integral propagator based solely on the above short-time propagator follows for any representation by concatenating Eq. (11) [or Eq. (13)] for a set of time subintervals $\left[t_{j}, t_{j+1}\right]$. In particular, to construct a path integral for the initial to the final coordinate we start with a given initial coordinate, switch to all intermediate initial momenta, and propagate within a single subinterval time to all intermediate final coordinates using Eq. (11). Repeating this propagation for all infinitesimal subintervals in the time partition $t_{0}, \ldots, t_{j}, t_{j+1}, \ldots, t$, with $t_{j+1}>t_{j}$, until the final prescribed time and coordinate gives

$$
\left\langle\mathbf{q}\left|\hat{U}\left(t, t_{0}\right)\right| \mathbf{q}_{0}\right\rangle=(2 \pi \hbar)^{-n N} \int \cdots \int d \mathbf{q}_{n-1} d \mathbf{p}_{n-1} \cdots d \mathbf{q}_{1} d \mathbf{p}_{1} d \mathbf{p}_{0} \prod_{j=0}^{n-1} \exp \left\{\frac{i}{\hbar}\left\{\phi\left(\mathbf{q}_{j+1}, \widetilde{\mathbf{q}}_{j} ; t_{j+1}, t_{j}\right)+\mathbf{p}_{j} \cdot\left[\widetilde{\mathbf{q}}_{j}-\mathbf{q}_{j}\right]\right\}\right\},
$$

with $\mathbf{q}_{n} \equiv \mathbf{q}$, initial time $t_{0}$, and final time $t_{n} \equiv t$; the limit $\left(t_{j+1}-t_{j}\right) \rightarrow 0 \quad(n \rightarrow \infty)$ is implied throughout. Similar integral forms follow for all other representations. For semiclassical purposes it is important to emphasize that this pathintegral formula is uniformly asymptotic. As mentioned for the above short-time form, Eq. (14) also turns out to be exact for most Hamiltonian operator forms. This path-integral form involves purely classical quantities, a useful feature that we exploit below. It may be contrasted with the more usual, and less general, coordinate space path-integral expressions [11] to which it is equivalent when $\partial_{\mathbf{p}}^{2} H$ is a positive-definite constant.

We now apply SSP to the path integral in Eq. (14). In particular, we do the integrals in the sequence of ordered pairs $\mathbf{p}_{j}$ followed by $\mathbf{q}_{j}$ for all subintervals, with $j=1, \ldots, n-1$; we need only do one SSP integral and the overall sequence of SSP integrals follows by induction. This approach, as we shall see below, leads to general properties for the indices and gives the complete stationary phase approximation to the overall path integral. The procedure is similar to our recent derivation [5]; however, here we begin with an alternate path-integral expression and specifically emphasize that the SSP is the only tool needed to obtain the result. Hence we only need evaluate the integral

$$
\begin{aligned}
\left\langle\mathbf{q}_{j+1}\left|\hat{U}\left(t_{j+1}, t_{0}\right)\right| \mathbf{q}_{0}\right\rangle^{\mathrm{sc}} \sim & \int d \mathbf{q}_{j}\left[\int d \mathbf{p}_{j}\left\langle\mathbf{q}_{j+1}\left|\hat{U}\left(t_{j+1}, t_{j}\right)\right| \mathbf{p}_{j}\right\rangle\right. \\
& \left.\times\left\langle\mathbf{p}_{j} \mid \mathbf{q}_{j}\right\rangle\right]\left\langle\mathbf{q}_{j}\left|\hat{U}\left(t_{j}, t_{0}\right)\right| \mathbf{q}_{0}\right\rangle^{\mathrm{sc}}
\end{aligned}
$$

by stationary phase, where for every given $j \geqslant 1$ the induction assumption is

$$
\begin{aligned}
\left\langle\mathbf{q}_{j}\left|\hat{U}\left(t_{j}, t_{0}\right)\right| \mathbf{q}_{0}\right\rangle^{\mathrm{sc}}= & (2 \pi i \hbar)^{-N / 2}\left|\operatorname{det} \partial_{\mathbf{p}_{0}} \mathbf{q}\left(\mathbf{q}_{0}, \mathbf{p}_{0} ; t_{j}, t_{0}\right)\right|^{-1 / 2} \\
& \times \exp \left(\frac{i}{\hbar} \phi\left(\mathbf{q}_{j}, \mathbf{q}_{0} ; t_{j}, t_{0}\right)\right. \\
& \left.-i \frac{\pi}{2} \nu\left[\mathbf{q}_{j}, \mathbf{q}_{0} ; t_{j}, t_{0}\right]\right)
\end{aligned}
$$

Here SC stands for semiclassical approximation. In Eq. (16) a finite sum is implied over all separate root trajectories, which can be labeled by a superscript $(k)$, i.e., all $\mathbf{p}\left(t_{0}\right)=\mathbf{p}_{0}=\mathbf{p}_{0}^{(k)} \quad$ given implicitly by $\mathbf{q}(t)=\mathbf{q}\left(\mathbf{q}_{0}, \mathbf{p}_{0} ; t_{j}, t_{0}\right)=\mathbf{q}_{j}$ or explicitly using the first relation in Eqs. (B2) for all functions $\phi=\phi^{(k)}$. The amplitude term in Eq. (16) has been rewritten in terms of a stability matrix, but can be written explicitly as function of $\mathbf{q}_{j}, \mathbf{q}_{0}$ in terms of $\partial_{\mathbf{q}_{j}} \partial_{\mathbf{q}_{0}} \phi$ by simply using the first identity in Eqs. (B3).

One remark on notation is in order. We denote a particular index for a propagator matrix element by $\nu[]$, where the argument within the square brackets denotes the particular representation being considered. For instance, in Eq. (16) the index along with the classical action generator $\phi$ are those for a given initial to final coordinate representation of the propagator within given end-point times.

Equation (16) is true for $t_{j} \rightarrow t_{0}^{+} \quad\left(=t_{0}+\epsilon, \epsilon>0\right.$ and small) since this is precisely the form taken by the short-time propagator for initial to final coordinate by doing stationary phase on the $\mathbf{p}_{0}$ to $\mathbf{q}_{0}$ transformation integral [doing only the first integral in Eq. (14)] while using Eq. (11). Indeed this is readily shown to give the initial trajectory index in Eq. (16) for $t-t_{0} \rightarrow 0^{+}$as

$$
\begin{aligned}
\nu\left[\mathbf{q}\left(t_{0}^{+}\right), \mathbf{q}_{0} ; t_{0}^{+}, t_{0}\right] & =\frac{1}{2}\left[\operatorname{sig}\left\{\left[\partial_{\mathbf{q}_{0}} \mathbf{q}\left(t_{0}^{+}\right)\right]^{-1} \partial_{\mathbf{p}_{0}} \mathbf{q}\left(t_{0}^{+}\right)\right\}-N\right] \\
& =\frac{1}{2}\left[\operatorname{sig} \partial_{\mathbf{p}_{0}} \mathbf{q}\left(t_{0}^{+}\right)-N\right]
\end{aligned}
$$

which is zero for cases in which $\partial_{\mathbf{p}}^{2} H$ is positive definite. Note that the initial index can also be reexpressed using the identities in Eq. (C9).

Putting Eq. (16) into Eq. (15) gives

$$
\begin{aligned}
& \left\langle\mathbf{q}_{j+1}\left|\hat{U}\left(t_{j+1}, t_{0}\right)\right| \mathbf{q}_{0}\right\rangle^{\mathrm{sc}} \\
& \sim \frac{(2 \pi i \hbar)^{-N / 2}}{(2 \pi \hbar)^{N}} \int d \mathbf{q}_{j} \int d \mathbf{p}_{j}\left|\operatorname{det} \partial_{\mathbf{p}_{0}} \mathbf{q}\left(\mathbf{q}_{0}, \mathbf{p}_{0} ; t_{j}, t_{0}\right)\right|^{-1 / 2} \\
& \quad \times \exp \left\{\frac { i } { \hbar } \left\{\phi\left(\mathbf{q}_{j+1}, \widetilde{\mathbf{q}}_{j} ; t_{j+1}, t_{j}\right)+\phi\left(\mathbf{q}_{j}, \mathbf{q}_{0} ; t_{j}, t_{0}\right)\right.\right. \\
& \left.\left.\quad+\mathbf{p}_{j} \cdot\left[\widetilde{\mathbf{q}}_{j}-\mathbf{q}_{j}\right]\right\}-i \frac{\pi}{2} \nu\left[\mathbf{q}_{j}, \mathbf{q}_{0} ; t_{j}, t_{0}\right]\right\}
\end{aligned}
$$


which is an integral of the form in Eq. (1) where we can identify $\mathbf{x} \equiv \mathbf{q}_{j}$ and $\mathbf{y} \equiv \mathbf{p}_{j}, \quad \varphi\left(\mathbf{q}_{j}, \mathbf{p}_{j} ; \boldsymbol{\alpha}\right)$ $\equiv \phi\left(\mathbf{q}_{j+1}, \widetilde{\mathbf{q}}_{j} ; t_{j+1}, t_{j}\right)+\phi\left(\mathbf{q}_{j}, \mathbf{q}_{0} ; t_{j}, t_{0}\right)+\mathbf{p}_{j} \cdot\left[\widetilde{\mathbf{q}}_{j}-\mathbf{q}_{j}\right]$, with the space of parameters $\boldsymbol{\alpha}=\left(\mathbf{q}_{j+1}, \mathbf{q}_{0}, t_{j+1}, t_{j}, t_{0}\right)$. We now apply the SSP formula [Eq. (10)] to Eq. (18). The stationaryphase condition for the $\mathbf{p}_{j}$ integral is [Eq. (4)]

$$
\begin{aligned}
\partial_{\mathbf{p}_{j}} \varphi & =\left[\partial_{\mathbf{q}_{j}} \phi\left(\mathbf{q}_{j+1}, \widetilde{\mathbf{q}}_{j} ; t_{j+1}, t_{j}\right)+\mathbf{p}_{j}\right] \cdot \partial_{\mathbf{p}_{j}} \widetilde{\mathbf{q}}_{j}+\widetilde{\mathbf{q}}_{j}-\mathbf{q}_{j} \\
& \equiv \widetilde{\mathbf{q}}_{j}-\mathbf{q}_{j}=\mathbf{0},
\end{aligned}
$$

where Eq. (12) has been used. This stationary-phase condition therefore gives stationary phase points $\mathbf{p}_{j}$ as function of $\mathbf{q}_{j}$ given by Eq. (12) with $\widetilde{\mathbf{q}}_{j}=\mathbf{q}_{j}$. Using Eqs. (12) and (19), the sub-Hessian at $\mathbf{p}_{j}=-\partial_{\mathbf{q}_{j}} \phi\left(\mathbf{q}_{j+1}, \mathbf{q}_{j} ; t_{j+1}, t_{j}\right)$ is then

$$
\begin{aligned}
\partial_{\mathbf{p}_{j}}^{2} \varphi & =\partial_{\mathbf{p}_{j}} \widetilde{\mathbf{q}}_{j}=-\left[\partial_{\mathbf{q}_{j}}^{2} \phi\left(\mathbf{q}_{j+1}, \mathbf{q}_{j} ; t_{j+1}, t_{j}\right)\right]^{-1} \\
& =-\left[\partial_{\mathbf{q}_{j}} \mathbf{q}\left(\mathbf{q}_{j}, \mathbf{p}_{j} ; t_{j+1}, t_{j}\right)\right]^{-1} \partial_{\mathbf{p}_{j}} \mathbf{q}\left(\mathbf{q}_{j}, \mathbf{p}_{j} ; t_{j+1}, t_{j}\right) .
\end{aligned}
$$

In the latter expression the second identity in Eqs. (B3) was used. Using Eq. (19), the phase function [e.g., $\widetilde{\varphi}(\mathbf{x})$ of Eq. (6)] becomes $\widetilde{\varphi}=\phi\left(\mathbf{q}_{j+1}, \mathbf{q}_{j} ; t_{j+1}, t_{j}\right)+\phi\left(\mathbf{q}_{j}, \mathbf{q}_{0} ; t_{j}, t_{0}\right)$. The stationary-phase condition for the remaining $\mathbf{q}_{j}$ integral is [e.g., Eq. (6)]

$$
\begin{aligned}
\left.\partial_{\mathbf{q}_{j}} \widetilde{\varphi}\right|_{\mathbf{q}_{j}=\mathbf{q}_{j}^{(k)}}= & {\left[\partial_{\mathbf{q}_{j}} \phi\left(\mathbf{q}_{j+1}, \mathbf{q}_{j} ; t_{j+1}, t_{j}\right)\right.} \\
& \left.+\partial_{\mathbf{q}_{j}} \phi\left(\mathbf{q}_{j}, \mathbf{q}_{0} ; t_{j}, t_{0}\right)\right]_{\mathbf{q}_{j}=\mathbf{q}_{j}^{(k)}} \\
\equiv & \left.\partial_{\mathbf{q}_{j}} \phi\left(\mathbf{q}_{j}, \mathbf{q}_{0} ; t_{j}, t_{0}\right)\right|_{\mathbf{q}_{j}=\mathbf{q}_{j}^{(k)}-\mathbf{p}_{j}^{(k)}=\mathbf{0} .}
\end{aligned}
$$

We have denoted the stationary-phase points with superscript labels, e.g., $\left(\mathbf{q}_{j}^{(k)}, \mathbf{p}_{j}^{(k)}\right)$. The labeling ( $k$ ) here is understood to correspond to a new set of root trajectories given by combining Eqs. (12), (19), and and (21). Indeed, upon using the second generator relation in Eq. (B2) and $\mathbf{q}\left(\mathbf{q}_{0}, \mathbf{p}_{0}^{(k)} ; t_{j}, t_{0}\right)=\mathbf{q}_{j}^{(k)}$, then $\mathbf{p}\left(\mathbf{q}_{0}, \mathbf{p}_{0}^{(k)} ; t_{j}, t_{0}\right)=\mathbf{p}_{j}^{(k)}$ and $\mathbf{q}\left(\mathbf{q}_{j}^{(k)}, \mathbf{p}_{j}^{(k)} ; t_{j+1}, t_{j}\right)=\mathbf{q}\left(\mathbf{q}_{0}, \mathbf{p}_{0}^{(k)} ; t_{j+1}, t_{0}\right)=\mathbf{q}_{j+1}$. Hence, at every stationary-phase point the phase is $\varphi\left(\mathbf{q}_{j}^{(k)}, \mathbf{p}_{j}^{(k)}\right)=\phi\left(\mathbf{q}_{j+1}, \mathbf{q}_{j}^{(k)} ; t_{j+1}, t_{j}\right)+\phi\left(\mathbf{q}_{j}^{(k)}, \mathbf{q}_{0} ; t_{j}, t_{0}\right)$, which, using Eq. (B1), is also equal to $\phi^{(k)}\left(\mathbf{q}_{j+1}, \mathbf{q}_{0} ; t_{j+1}, t_{0}\right)$, the classical action for every root trajectory satisfying $\mathbf{q}\left(\mathbf{q}_{0}, \mathbf{p}_{0}^{(k)} ; t_{j+1}, t_{0}\right)=\mathbf{q}_{j+1}$.

It remains to determine $\partial_{\mathbf{q}_{j}}^{2} \widetilde{\varphi}$. This is given by differentiating the expression in Eq. (21) with respect to $\mathbf{q}_{j}$ and using the third identity in Eqs. (B3). Equivalently, we use the analog of Eq. (A2) while using Eq. (20), $\partial_{\mathbf{q}_{j}} \partial_{\mathbf{p}_{j}} \varphi$ $=\mathbf{I}_{N}$, and the third identity in Eqs. (B3), $\partial_{\mathbf{q}_{j}}^{2} \varphi=\partial_{\mathbf{q}_{j}}^{2} \phi\left(\mathbf{q}_{j}, \mathbf{q}_{0} ; t_{j}, t_{0}\right)=\partial_{\mathbf{p}_{0}} \mathbf{p}\left(t_{j}\right)\left[\partial_{\mathbf{p}_{0}} \mathbf{q}\left(t_{j}\right)\right]^{-1}$; then

$$
\begin{aligned}
\partial_{\mathbf{q}_{j}}^{2} \widetilde{\varphi}= & \partial_{\mathbf{q}_{j}}^{2} \varphi-\partial_{\mathbf{p}_{j}} \partial_{\mathbf{q}_{j}} \varphi \cdot\left[\partial_{\mathbf{p}_{j}}^{2} \varphi\right]^{-1} \cdot\left[\partial_{\mathbf{p}_{j}} \partial_{\mathbf{q}_{j}} \varphi\right]^{T} \\
= & \partial_{\mathbf{p}_{0}} \mathbf{p}\left(t_{j}\right) \cdot\left[\partial_{\mathbf{p}_{0}} \mathbf{q}\left(t_{j}\right)\right]^{-1} \\
& +\left[\partial_{\mathbf{p}_{j}} \mathbf{q}\left(\mathbf{q}_{j}, \mathbf{p}_{j} ; t_{j+1}, t_{j}\right)\right]^{-1} \cdot \partial_{\mathbf{q}_{j}} \mathbf{q}\left(\mathbf{q}_{j}, \mathbf{p}_{j} ; t_{j+1}, t_{j}\right) .
\end{aligned}
$$

(Note: For compactness, the explicit dependence on initial conditions $\mathbf{q}_{0}, \mathbf{p}_{0}, t_{0}$ is dropped for the coordinate and momentum of any trajectory beginning at $t_{0}$.) We can now rewrite this expression by using the composite function property for any classical trajectory: $\mathbf{q}\left(t_{j+1}\right)$ $=\mathbf{q}\left(\mathbf{q}_{j}, \mathbf{p}_{j} ; t_{j+1}, t_{j}\right) \equiv \mathbf{q}\left(\mathbf{q}\left(t_{j}\right), \mathbf{p}\left(t_{j}\right) ; t_{j+1}, t_{j}\right)$. Differentiating this with respect to $\mathbf{p}_{0}$ (for fixed $\mathbf{q}_{0}$ and time variables) gives

$$
\begin{aligned}
\partial_{\mathbf{p}_{0}} \mathbf{q}\left(t_{j+1}\right)= & \partial_{\mathbf{q}_{j}} \mathbf{q}\left(\mathbf{q}_{j}, \mathbf{p}_{j} ; t_{j+1}, t_{j}\right) \cdot \partial_{\mathbf{p}_{0}} \mathbf{q}\left(t_{j}\right) \\
& +\partial_{\mathbf{p}_{j}} \mathbf{q}\left(\mathbf{q}_{j}, \mathbf{p}_{j} ; t_{j+1}, t_{j}\right) \cdot \partial_{\mathbf{p}_{0}} \mathbf{p}\left(t_{j}\right) .
\end{aligned}
$$

Multiplying this equation by $\left[\partial_{\mathbf{p}_{j}} \mathbf{q}\left(\mathbf{q}_{j}, \mathbf{p}_{j} ; t_{j+1}, t_{j}\right)\right]^{-1}$ on the left and by $\left[\partial_{\mathbf{p}_{0}} \mathbf{q}\left(t_{j}\right)\right]^{-1}$ on the right then also gives the identity

$$
\begin{aligned}
& \partial_{\mathbf{p}_{0}} \mathbf{p}\left(t_{j}\right) \cdot\left[\partial_{\mathbf{p}_{0}} \mathbf{q}\left(t_{j}\right)\right]^{-1} \\
&+ {\left[\partial_{\mathbf{p}_{j}} \mathbf{q}\left(\mathbf{q}_{j}, \mathbf{p}_{j} ; t_{j+1}, t_{j}\right)\right]^{-1} \partial_{\mathbf{q}_{j}} \mathbf{q}\left(\mathbf{q}_{j}, \mathbf{p}_{j} ; t_{j+1}, t_{j}\right) } \\
& \quad=\left[\partial_{\mathbf{p}_{j}} \mathbf{q}\left(\mathbf{q}_{j}, \mathbf{p}_{j} ; t_{j+1}, t_{j}\right)\right]^{-1} \cdot \partial_{\mathbf{p}_{0}} \mathbf{q}\left(t_{j+1}\right) \cdot\left[\partial_{\mathbf{p}_{0}} \mathbf{q}\left(t_{j}\right)\right]^{-1} .
\end{aligned}
$$

Hence Eq. (22) also reads

$$
\partial_{\mathbf{q}_{j}}^{2} \widetilde{\varphi}=\left[\partial_{\mathbf{p}_{j}} \mathbf{q}\left(\mathbf{q}_{j}, \mathbf{p}_{j} ; t_{j+1}, t_{j}\right)\right]^{-1} \cdot \partial_{\mathbf{p}_{0}} \mathbf{q}\left(t_{j+1}\right) \cdot\left[\partial_{\mathbf{p}_{0}} \mathbf{q}\left(t_{j}\right)\right]^{-1}
$$

Finally, we can substitute the matrix determinants and signatures of $\partial_{\mathbf{p}_{j}}^{2} \varphi$ and $\partial_{\mathbf{q}_{j}}^{2} \tilde{\varphi}$ obtained from Eqs. (20) and (23) into the SSP formula of Eq. (24) below. Before doing so we make note of a subtle point concerning the sub-Hessian condition. That is, according to Eq. (20), the relevant subHessian condition that allows the use of sequential stationary phase is $\operatorname{det} \partial_{\mathbf{p}_{j}} \mathbf{q}\left(\mathbf{q}_{j}, \mathbf{p}_{j} ; t_{j+1}, t_{j}\right) \neq 0$, in which we have used the fact that the local stability matrix $\partial_{\mathbf{q}_{j}} \mathbf{q}\left(\mathbf{q}_{j}, \mathbf{p}_{j} ; t_{j+1}, t_{j}\right)$ is positive definite and approaches $\mathbf{I}_{N}$ within the limit $t_{j+1}-t_{j} \rightarrow 0$. This sub-Hessian condition can always be satisfied. Indeed, the trajectories and classical stability matrix elements are analytic functions of their arguments and hence along any trajectory, the value of the function $\operatorname{det} \partial_{\mathbf{p}_{j}} \mathbf{q}\left(\mathbf{q}_{j}, \mathbf{p}_{j} ; \tau, t_{j}\right)$ can be zero only at a finite number of measure zero points. Thus there always exist time partitions $t_{j}$ for which all the above stability matrices are nonsingular at those times as long as the end-point times $t_{0}, t$ are nonfocal. That is, only a finite number of focal or caustic points exist within any trajectory segment unless the determinant function is identically zero (a trivial nonphysical case). It is hence to be understood that all phase-space endpoints used in all index equations are unequal to focal points where the stability matrices occurring in the equations would otherwise be singular and the indices simply undefined.

Now using Eqs. (20) and (23) in the SSP formula 


$$
\begin{aligned}
\left\langle\mathbf{q}_{j+1}\right| & \hat{U}\left(t_{j+1}, t_{0}\right)\left|\mathbf{q}_{0}\right\rangle^{\mathrm{sc}} \\
= & (2 \pi i \hbar)^{-N / 2}\left|\operatorname{det} \partial_{\mathbf{p}_{0}} \mathbf{q}\left(t_{j}\right) \operatorname{det} \partial_{\mathbf{p}_{j}}^{2} \varphi \operatorname{det} \partial_{\mathbf{q}_{j}}^{2} \widetilde{\varphi}\right|^{-1 / 2} \\
& \times \exp \left\{\frac{i}{\hbar} \phi\left(\mathbf{q}_{j+1}, \mathbf{q}_{0} ; t_{j+1}, t_{0}\right)-i \frac{\pi}{2}\left\{\nu\left[\mathbf{q}_{j}, \mathbf{q}_{0} ; t_{j}, t_{0}\right]\right.\right. \\
& \left.\left.-\frac{1}{2}\left[\operatorname{sig} \partial_{\mathbf{p}_{j}}^{2} \varphi+\operatorname{sig} \partial_{\mathbf{q}_{j}}^{2} \tilde{\varphi}\right]\right\}\right\}
\end{aligned}
$$

gives

$$
\begin{aligned}
\left\langle\mathbf{q}_{j+1}\left|\hat{U}\left(t_{j+1}, t_{0}\right)\right| \mathbf{q}_{0}\right\rangle^{\mathrm{sc}} & \\
= & (2 \pi i \hbar)^{-N / 2}\left|\operatorname{det} \partial_{\mathbf{p}_{0}} \mathbf{q}\left(t_{j+1}\right)\right|^{-1 / 2} \\
& \times \exp \left(\frac{i}{\hbar} \phi\left(\mathbf{q}_{j+1}, \mathbf{q}_{0} ; t_{j+1}, t_{0}\right)\right. \\
& \left.-i \frac{\pi}{2} \nu\left[\mathbf{q}_{j+1}, \mathbf{q}_{0} ; t_{j+1}, t_{0}\right]\right) .
\end{aligned}
$$

This is of the same form as the induction Eq. (16), for $j \rightarrow j+1$, again with implied sum over all $\mathbf{p}_{0}=\mathbf{p}_{0}^{(k)}$ and actions $\phi=\phi^{(k)}$, i.e., all root trajectories now satisfying $\mathbf{q}\left(t_{0}\right)=\mathbf{q}_{0}$ and $\mathbf{q}\left(t_{j+1}\right)=\mathbf{q}_{j+1}$. The index in Eq. (25) is given by

$\nu\left[\mathbf{q}_{j+1}, \mathbf{q}_{0} ; t_{j+1}, t_{0}\right]=\nu\left[\mathbf{q}_{j}, \mathbf{q}_{0} ; t_{j}, t_{0}\right]+\frac{1}{2}\left(\mu_{j}-\sigma_{j}\right)$,

where the integer quantities $\mu_{j}$ and $\sigma_{j}$ are given by the equivalent expressions

$$
\begin{aligned}
\mu_{j} & =\operatorname{sig}\left\{\left[\partial_{\mathbf{q}_{j}} \mathbf{q}\left(\mathbf{q}_{j}, \mathbf{p}_{j} ; t_{j+1}, t_{j}\right)\right]^{-1} \partial_{\mathbf{p}_{j}} \mathbf{q}\left(\mathbf{q}_{j}, \mathbf{p}_{j} ; t_{j+1}, t_{j}\right)\right\} \\
& =\operatorname{sig}\left\{\left[\partial_{\mathbf{p}_{j}} \mathbf{q}\left(\mathbf{q}_{j}, \mathbf{p}_{j} ; t_{j+1}, t_{j}\right)^{-1} \partial_{\mathbf{q}_{j}} \mathbf{q}\left(\mathbf{q}_{j}, \mathbf{p}_{j} ; t_{j+1}, t_{j}\right)\right]\right\} \\
& =\operatorname{sig} \partial_{\mathbf{p}_{j}} \mathbf{q}\left(\mathbf{q}_{j}, \mathbf{p}_{j} ; t_{j+1}, t_{j}\right) \equiv \operatorname{sig} \mathbf{A}\left(t_{j+1}, t_{j}\right)^{-1} \mathbf{B}\left(t_{j+1}, t_{j}\right) \\
& =\operatorname{sig} \mathbf{B}\left(t_{j+1}, t_{j}\right)^{-1} \mathbf{A}\left(t_{j+1}, t_{j}\right)
\end{aligned}
$$

and

$$
\begin{aligned}
\sigma_{j} & =\operatorname{sig}\left\{\left[\partial_{\mathbf{p}_{j}} \mathbf{q}\left(\mathbf{q}_{j}, \mathbf{p}_{j} ; t_{j+1}, t_{j}\right)\right]^{-1} \partial_{\mathbf{p}_{0}} \mathbf{q}\left(t_{j+1}\right)\left[\partial_{\mathbf{p}_{0}} \mathbf{q}\left(t_{j}\right)\right]^{-1}\right\} \\
& =\operatorname{sig}\left\{\partial_{\mathbf{p}_{0}} \mathbf{q}\left(t_{j}\right)\left[\partial_{\mathbf{p}_{0}} \mathbf{q}\left(t_{j+1}\right)\right]^{-1} \partial_{\mathbf{p}_{j}} \mathbf{q}\left(\mathbf{q}_{j}, \mathbf{p}_{j} ; t_{j+1}, t_{j}\right)\right\} \\
& \equiv \operatorname{sig}\left\{\mathbf{B}\left(t_{j+1}, t_{j}\right)^{-1} \mathbf{B}\left(t_{j+1}\right) \mathbf{B}\left(t_{j}\right)^{-1}\right\} \\
& =\operatorname{sig}\left\{\mathbf{B}\left(t_{j}\right) \mathbf{B}\left(t_{j+1}\right)^{-1} \mathbf{B}\left(t_{j+1}, t_{j}\right)\right\}
\end{aligned}
$$

The second expression for $\sigma_{j}$ arises from the property $\operatorname{sig} \mathbf{S}=\operatorname{sig} \mathbf{S}^{-1}$ for any nonsingular symmetric matrix $\mathbf{S}$. The last equality on the second line of Eq. (27) follows in the limit $t_{j+1}-t_{j} \rightarrow 0$ since $\partial_{\mathbf{q}_{j}} \mathbf{q}\left(\mathbf{q}_{j}, \mathbf{p}_{j} ; t_{j+1}, t_{j}\right) \rightarrow \mathbf{I}_{N}$, whereas the second equality follows from the general properties in Eqs. (C6) and (C9). Note also that we have reexpressed the index property using the compact matrix notation of Appendix $\mathrm{C}$ and that the local stability matrices are also expressed in compact notation.

Equation (26) therefore gives a general infinitesimal additive property of the index. It expresses, in algebraic form, the change in the trajectory's index due to its further evolution, over an infinitesimal time $t_{j+1}-t_{j}$, from any phase-space end point $\left(\mathbf{q}_{j}, \mathbf{p}_{j}\right)$ at time $t_{j} \neq t_{0}$ to an end point $\left(\mathbf{q}_{j+1}, \mathbf{p}_{j+1}\right)$ at time $t_{j+1}$. By induction, we see that these subinterval contributions are added up, via Eq. (26), together with the nonzero initial index given in Eq. (17). Hence the SSP process proves that the index for a total time interval $\left[t_{0}, t\right]$ is given by

$$
\nu\left[\mathbf{q}, \mathbf{q}_{0} ; t, t_{0}\right]=\nu\left[\mathbf{q}\left(t_{0}^{+}\right), \mathbf{q}_{0} ; t_{0}^{+}, t_{0}\right]+\frac{1}{2} \sum_{j}\left(\mu_{j}-\sigma_{j}\right) .
$$

We note here, as is shown immediately below, that Eq. (26), and hence Eq. (29), holds for sufficiently small, yet finite, time segments $\left[t_{j}, t_{j+1}\right]$. This property turns out to be a useful one for computational purposes, as discussed briefly below. Based on Eqs. (25)-(28) and induction, the above SSP proof therefore gives the (nonuniform at caustics) semiclassical propagator for the initial to final coordinate representation in familiar form $[2,3,5,13]$

$$
\begin{aligned}
\left\langle\mathbf{q}\left|\hat{U}\left(t, t_{0}\right)\right| \mathbf{q}_{0}\right\rangle^{\mathrm{sc}}= & (2 \pi i \hbar)^{-N / 2}\left|\operatorname{det} \partial_{\mathbf{p}_{0}} \mathbf{q}(t)\right|^{-1 / 2} \\
& \times \exp \left[\frac{i}{\hbar} \phi\left(\mathbf{q}, \mathbf{q}_{0} ; t, t_{0}\right)-i \frac{\pi}{2} \nu\left[\mathbf{q}, \mathbf{q}_{0} ; t, t_{0}\right]\right],
\end{aligned}
$$

with, as always, an implied sum over similar terms for every trajectory satisfying two-point coordinate boundary value conditions: $\mathbf{q}(t) \equiv \mathbf{q}\left(\mathbf{q}_{0}, \mathbf{p}_{0} ; t, t_{0}\right)=\mathbf{q}$. Here, however, in contrast to earlier developments, we are dealing with more general $H$ forms and our approach allows us to readily develop additional index properties.

These additional index properties follow in a simple manner. For instance, Eq. (28) readily gives the known result that within any time interval a change in the trajectory index can only occur if a focal point lies within the interval, since otherwise $\partial_{\mathbf{p}_{0}} \mathbf{q}\left(t_{j+1}\right)\left[\partial_{\mathbf{p}_{0}} \mathbf{q}\left(t_{j}\right)\right]^{-1} \rightarrow \mathbf{I}_{N}$, giving $\sigma_{j}=\mu_{j}$; from Eq. (26) we would obtain $\nu\left[\mathbf{q}_{j+1}, \mathbf{q}_{0} ; t_{j+1}, t_{0}\right]$ $=\nu\left[\mathbf{q}_{j}, \mathbf{q}_{0} ; t_{j}, t_{0}\right]$. As usual, these focal points are defined by singularities in the matrix inverse or zero determinants of $\partial_{\mathbf{p}_{0}} \mathbf{q}(\tau)$ existing at focal times denoted by $\tau=t_{i}^{*}>t_{0}$. The index for any given trajectory can therefore be written in terms of a sum over index changes due to all such possibly encountered focal points:

$$
\begin{aligned}
\nu\left[\mathbf{q}, \mathbf{q}_{0} ; t, t_{0}\right]= & \frac{1}{2}\left[\operatorname{sig} \partial_{\mathbf{p}_{0}} \mathbf{q}\left(t_{0}^{+}\right)-N\right] \\
& +\frac{1}{2} \sum_{i}\left[\operatorname{sig} \partial_{\mathbf{p}_{i}^{*}} \mathbf{q}\left(\mathbf{q}_{i}^{*}, \mathbf{p}_{i}^{*} ; t_{i}^{*}+\epsilon, t_{i}^{*}-\epsilon\right)-\sigma_{i}^{*}\right]
\end{aligned}
$$

where

$$
\begin{aligned}
\sigma_{i}^{*}= & \operatorname{sig}\left\{\partial_{\mathbf{p}_{0}} \mathbf{q}\left(t_{i}^{*}-\boldsymbol{\epsilon}\right)\left[\partial_{\mathbf{p}_{0}} \mathbf{q}\left(t_{i}^{*}+\boldsymbol{\epsilon}\right)\right]^{-1}\right. \\
& \left.\times \partial_{\mathbf{p}_{i}^{*}} \mathbf{q}\left(\mathbf{q}_{i}^{*}, \mathbf{p}_{i}^{*} ; t_{i}^{*}+\boldsymbol{\epsilon}, t_{i}^{*}-\boldsymbol{\epsilon}\right)\right\},
\end{aligned}
$$

and $\mathbf{q}_{i}^{*}=\mathbf{q}\left(t_{i}^{*}-\boldsymbol{\epsilon}\right), \mathbf{p}_{i}^{*}=\mathbf{p}\left(t_{i}^{*}-\boldsymbol{\epsilon}\right)$, and the limit $\epsilon \rightarrow 0^{+}$is implied, where + denotes approaching 0 from above. 
Before displaying other properties that result from SSP operations, we note that for Hamiltonians with $\partial_{\mathbf{p}}^{2} H=\mathbf{m}^{-1}$ (or positive-definite constant matrix) these index formulas can be further recast by taking into account the short-time stability matrix property stated just above Eq. (24). This allows us to put, for the local stability matrices, $\quad \operatorname{sig} \partial_{\mathbf{p}_{j}} \mathbf{q}\left(\mathbf{q}_{j}, \mathbf{p}_{j} ; t_{j+1}, t_{j}\right)=\mu_{j}=N$ and then $\sigma_{j}=\operatorname{sig}\left\{\partial_{\mathbf{p}_{0}} \mathbf{q}\left(t_{j+1}\right)\left[\partial_{\mathbf{p}_{0}} \mathbf{q}\left(t_{j}\right)\right]^{-1}\right\} \quad$ or $\quad \sigma_{i}^{*}=\operatorname{sig}\left\{\partial_{\mathbf{p}_{0}} \mathbf{q}\left(t_{i}^{*}\right.\right.$ $\left.+\epsilon)\left[\partial_{\mathbf{p}_{0}} \mathbf{q}\left(t_{i}^{*}-\epsilon\right)\right]^{-1}\right\}$, thereby recovering from the above equations the previously derived result [2,5,13] $\nu\left[\mathbf{q}, \mathbf{q}_{0} ; t, t_{0}\right]=\Sigma_{j}\left(N-\sigma_{j}\right) / 2$, in which the initial index is zero. This is also expressible as the count of multiplicities of zeros of the stability matrix $\partial_{\mathbf{p}_{0}} \mathbf{q}(\tau)$ encountered along the course of the trajectory [3]. It is to be noted, as will become clear, that Eq. (31) is not as simple and practically useful as is the straightforward application of Eq. (29) to small finite segments.

We can now extract a further index property that is useful and less well known. This is a property for adding up or "patching together" indices belonging to any two joining finite trajectory segments (i.e., segments that together form an overall trajectory) to give the index for an overall trajectory. Consider an interval $\left[t_{0}, t_{j+1}\right]$ in which a trajectory begins with any coordinate $\mathbf{q}\left(t_{0}\right)=\mathbf{q}_{0}$ (and given momentum $\left.\mathbf{p}_{0}\right)$ and ends with coordinate $\mathbf{q}\left(t_{j+1}\right)=\mathbf{q}_{j+1}$. As shown above, the index $\nu\left[\mathbf{q}_{j+1}, \mathbf{q}_{0} ; t_{j+1}, t_{0}\right]$ for any such trajectory is generally given by Eq. (29) with $\mathbf{q}=\mathbf{q}_{j+1}, t=t_{j+1}$. Say we now divide up the trajectory for the total-time interval into two adjoining finite segments, each corresponding to a finitetime subinterval $\left[t_{0}, t_{j+1}\right]=\left[t_{0}, t_{j}\right]+\left[t_{j}, t_{j+1}\right]$, with all time end points being nonfocal. [Note that $t_{j+1}-t_{j}$ is now not necessarily infinitesimal, as it was in Eq. (26).] Now each trajectory segment, being a separate trajectory, has an index assigned to it. For the first segment, the index is $\nu\left[\mathbf{q}_{j}, \mathbf{q}_{0} ; t_{j}, t_{0}\right]$ with phase-space end point $\mathbf{q}\left(t_{j}\right)=\mathbf{q}_{j}$, $\mathbf{p}\left(t_{j}\right)=\mathbf{p}_{j}$, which is then considered as the initial point for the other joining trajectory segment with index $\nu\left[\mathbf{q}_{j+1}, \mathbf{q}_{j} ; t_{j+1}, t_{j}\right]$. We can now obtain a formula for adding up the indices for the separate segments to give the index for the total trajectory. This is easily done while proving a relation among the indices by considering the integral joining up the interval propagations,

$$
\begin{aligned}
\left\langle\mathbf{q}_{j+1}\right| & \left.\left|\hat{U}\left(t_{j+1}, t_{0}\right)\right| \mathbf{q}_{0}\right\rangle^{\mathrm{sc}} \\
& \sim \int d \mathbf{q}_{j}\left\langle\mathbf{q}_{j+1}\left|\hat{U}\left(t_{j+1}, t_{j}\right)\right| \mathbf{q}_{j}\right\rangle^{\mathrm{sc}}\left\langle\mathbf{q}_{j}\left|\hat{U}\left(t_{j}, t_{0}\right)\right| \mathbf{q}_{0}\right\rangle^{\mathrm{sc}} \\
& \sim(2 \pi i \hbar)^{-N} \int d \mathbf{q}_{j} \mid \operatorname{det} \partial_{\mathbf{p}_{0}} \mathbf{q}\left(t_{j}\right) \\
\quad & \times\left.\operatorname{det} \partial_{\mathbf{p}_{j}} \mathbf{q}\left(\mathbf{q}_{j}, \mathbf{p}_{j} ; t_{j+1}, t_{j}\right)\right|^{-1 / 2} \\
\quad & \times \exp \left\{\frac{i}{\hbar}\left[\phi\left(\mathbf{q}_{j+1}, \mathbf{q}_{j} ; t_{j+1}, t_{j}\right)+\phi\left(\mathbf{q}_{j}, \mathbf{q}_{0} ; t_{j}, t_{0}\right)\right]\right. \\
& \left.-i \frac{\pi}{2}\left(\nu\left[\mathbf{q}_{j+1}, \mathbf{q}_{j} ; t_{j+1}, t_{j}\right]+\nu\left[\mathbf{q}_{j}, \mathbf{q}_{0} ; t_{j}, t_{0}\right]\right)\right\},
\end{aligned}
$$

within the stationary-phase approximation. This operation must recover Eq. (30), for $t=t_{j+1}$, since it corresponds to another overall SSP operation on the path integral from time $t_{0}$ to $t_{j+1}$, only in a differently ordered fashion. The stationary-phase approximation to the above integral follows immediately since the analysis is similar to the $\mathbf{q}_{j}$ part of the integral already done for Eq. (18). The Hessian is given by the expressions in Eq. (23) or (22). By using it, the stationary-phase formula applied to Eq. (33) reduces to the required semiclassical form given by Eq. (30) with the index identity resulting:

$$
\begin{aligned}
\nu\left[\mathbf{q}_{j+1}, \mathbf{q}_{0} ; t_{j+1}, t_{0}\right]= & \nu\left[\mathbf{q}_{j+1}, \mathbf{q}_{j} ; t_{j+1}, t_{j}\right]+\nu\left[\mathbf{q}_{j}, \mathbf{q}_{0} ; t_{j}, t_{0}\right] \\
& +\frac{1}{2}\left(N-\sigma_{j}\right) .
\end{aligned}
$$

This is then a general prescription for computing the index, for the initial to final coordinate representation, using the separate indices, of the same kind, for any two finite joining trajectory segments. Equation (34) can be used when one has run a trajectory until an intermediate time $t_{j}$, computed its corresponding index using the general formula of Eq. (29), and then wishes to extend the computation of the index for the overall trajectory evolved for a further finitetime interval until time $t_{j+1}$ by treating the final intermediate end point as the initial phase-space point for the joining trajectory segment.

A very useful and simple property, however, obtains from Eq. (34) by considering the important situation in which $t_{j+1}-t_{j}$ is finite but sufficiently small, so that the local stability matrix $\partial_{\mathbf{q}_{j}} \mathbf{q}\left(\mathbf{q}_{j}, \mathbf{p}_{j} ; \tau, t_{j}\right)$ (which always begins as $\mathbf{I}_{N}$ for $\tau=t_{j}$ ) is nonsingular for all $\tau$ within $\left[t_{j}, t_{j+1}\right]$. In such a case Eq. (34) simplifies to Eq. (26). Thus Eq. (26) hence not only applies in the $t_{j+1}-t_{j} \rightarrow 0$ limit, but also applies to small finite segments. Let us first show this for the case where $\partial_{\mathbf{p}_{j}} \mathbf{q}\left(\mathbf{q}_{j}, \mathbf{p}_{j} ; \tau, t_{j}\right)$ is also nonsingular within the same interval. Since the latter stability matrix is nonsingular, then its signature does not change at any time within $\left[t_{j}, t_{j+1}\right]$, giving $\nu\left[\mathbf{q}_{j+1}, \mathbf{q}_{j} ; t_{j+1}, t_{j}\right]=\nu\left[\mathbf{q}\left(t_{j}^{+}\right), \mathbf{q}_{j} ; t_{j}^{+}, t_{j}\right]$ $=\frac{1}{2}\left[\operatorname{sig} \partial_{\mathbf{p}_{j}} \mathbf{q}\left(\mathbf{q}_{j}, \mathbf{p}_{j} ; t_{j}^{+}, t_{j}\right)-N\right]=\frac{1}{2}\left(\mu_{j}-N\right)$. Putting this into Eq. (34) shows that Eq. (26), and hence Eq. (29), is also true for sufficiently small, yet finite, time intervals $\left[t_{j}, t_{j+1}\right]$. To prove this property without having to assume another stability matrix condition follows by using one of the index "connecting relations" discussed in Sec. III B, namely, Eq. (54) with $t_{0}, t$ replaced by $t_{j}, t_{j+1}$, giving $\nu\left[\mathbf{q}_{j+1}, \mathbf{q}_{j} ; t_{j+1}, t_{j}\right]$ $=\nu\left[\mathbf{q}_{j+1}, \mathbf{p}_{j} ; t_{j+1}, t_{j}\right]+\frac{1}{2}\left(\mu_{j}-N\right)$. The $\nu\left[\mathbf{q}_{j+1}, \mathbf{p}_{j} ; t_{j+1}, t_{j}\right]$ index, discussed below, is that for the initial momentum to final coordinate representation and as seen from Eq. (11) has a value of zero for initial time $\tau=t_{j}$. Since this index changes only when $\partial_{\mathbf{q}_{j}} \mathbf{q}$ becomes singular within the interval then, by the above assumption, $\nu\left[\mathbf{q}_{j+1}, \mathbf{p}_{j} ; t_{j+1}, t_{j}\right]=0$, again proving the property.

Equation (26) then leads to a useful prescription for computing the index. Such prescriptions have been discussed recently [5], where it was shown that similar computations apply to the indices for the other representations. That is, numerical integration of Hamilton's equations plus the stability matrix equations [Eq. (C4)] is done using sufficiently small time steps, i.e., $\Delta t=t_{j+1}-t_{j}$. From the first small step $t_{0}$ to $t_{1}=t_{0}+\Delta$, we compute $\mu_{0}=\operatorname{sig}\left[\partial_{\mathbf{q}_{0}} \mathbf{q}\left(t_{1}\right)\right]^{-1} \partial_{\mathbf{p}_{0}} \mathbf{q}\left(t_{1}\right)$ 
or $\mu_{0}=\operatorname{sig} \partial_{\mathbf{p}_{0}} \mathbf{q}\left(t_{1}\right) \partial_{\mathbf{q}_{0}} \mathbf{q}\left(t_{1}\right)^{T} \equiv \operatorname{sig} \mathbf{B}\left(t_{1}\right) \mathbf{A}\left(t_{1}\right)^{T}$, which are equivalent and convenient ways of obtaining the initial index needed in Eq. (29) as $\frac{1}{2}\left(\mu_{0}-N\right)$. Then, beginning with $t_{1}$, for every trajectory time step we compute $\frac{1}{2}\left(\mu_{j}-\sigma_{j}\right)$ and add this integer to the overall index, where $\mu_{j}$ and $\sigma_{j}$ are given in Eqs. (27) and (28). The total index at time $t$ is then obtained by summing up the index changes for all interval steps as given by Eq. (29).

The analogs of Eqs. (26)-(30) and (34) for the other representations follow naturally by similar SSP treatments on the path-integral forms arising from the short-time formula of Eq. (11) or (13); hence we do not repeat all of the above analysis. Rather, we only explicitly derive the index properties analogous to Eqs. (26)-(30) and (34) for the other basic type of final coordinate representation: the initial momentum to final coordinate representation. The analysis used to arrive at the analogous equations for the matrix elements and the indices for the final momentum representations does not have to be repeated since all required formulas follow by the simple replacements $\mathbf{q} \rightarrow-\mathbf{p}$ and $\mathbf{p} \rightarrow \mathbf{q}$. This canonical transformation reverses the roles of coordinates and momenta. At the same time, what follows is a display of the inherent canonical structure of the index formulas.

The analog of Eq. (30) for the initial momentum to final coordinate representation takes the familiar nonuniform semiclassical propagator form

$$
\begin{aligned}
\left\langle\mathbf{q}\left|\hat{U}\left(t, t_{0}\right)\right| \mathbf{p}_{0}\right\rangle^{\mathrm{sc}} & \\
= & (2 \pi i \hbar)^{-N / 2}\left|\operatorname{det}_{\mathbf{q}_{0}} \mathbf{q}(t)\right|^{-1 / 2} \exp \left[\frac{i}{\hbar} \widetilde{\phi}\left(\mathbf{q}, \mathbf{p}_{0} ; t, t_{0}\right)\right. \\
& \left.-i \frac{\pi}{2} \nu\left[\mathbf{q}, \mathbf{p}_{0} ; t, t_{0}\right]\right],
\end{aligned}
$$

with implied sum over similar terms for all root trajectories: $\mathbf{q}(t)=\mathbf{q}$ and given $\mathbf{p}\left(t_{0}\right)=\mathbf{p}_{0}$. The properties for the index $\nu\left[\mathbf{q}, \mathbf{p}_{0} ; t, t_{0}\right]$ now follow by considering an integral that joins two finite propagations

$$
\begin{aligned}
& \left\langle\mathbf{q}_{j+1}\left|\hat{U}\left(t_{j+1}, t_{0}\right)\right| \mathbf{p}_{0}\right\rangle^{\mathrm{sc}} \sim \int d \mathbf{q}_{j}\left[\int d \mathbf{p}_{j}\left\langle\mathbf{q}_{j+1}\left|\hat{U}\left(t_{j+1}, t_{j}\right)\right| \mathbf{p}_{j}\right\rangle^{\mathrm{sc}}\left\langle\mathbf{p}_{j} \mid \mathbf{q}_{j}\right\rangle\right]\left\langle\mathbf{q}_{j}\left|\hat{U}\left(t_{j}, t_{0}\right)\right| \mathbf{p}_{0}\right)^{\mathrm{sc} \sim} \frac{(2 \pi i \hbar)^{-N / 2}}{(2 \pi \hbar)^{N}} \int d \mathbf{q}_{j} \\
& \quad \times \int d \mathbf{p}_{j}\left|\operatorname{det} \partial_{\mathbf{q}_{0}} \mathbf{q}\left(t_{j}\right) \operatorname{det} \partial_{\mathbf{q}_{j}} \mathbf{q}\left(\mathbf{q}_{j}, \mathbf{p}_{j} ; t_{j+1}, t_{j}\right)\right|^{-1 / 2} \exp \left[\frac{i}{\hbar}\left[\widetilde{\phi}\left(\mathbf{q}_{j+1}, \mathbf{p}_{j} ; t_{j+1}, t_{j}\right)+\widetilde{\phi}\left(\mathbf{q}_{j}, \mathbf{p}_{0} ; t_{j}, t_{0}\right)-\mathbf{q}_{j} \cdot \mathbf{p}_{j}\right]\right. \\
& \left.\quad-i \frac{\pi}{2}\left(\nu\left[\mathbf{q}_{j+1}, \mathbf{p}_{j} ; t_{j+1}, t_{j}\right]+\nu\left[\mathbf{q}_{j}, \mathbf{p}_{0} ; t_{j}, t_{0}\right]\right)\right],
\end{aligned}
$$

within the stationary-phase approximation. This is done using the SSP process where the steps are almost identical to Eqs. (19)-(23) by now using the identities in Appendix B for the generator $\widetilde{\phi}$ instead of those for $\phi$. Moreover, by using a version of the composite property given above Eq. (23) in which $\mathbf{p}_{0}$ is now replaced by $\mathbf{q}_{0}$, this finally leads to the property [cf. Eq. (34)]

$$
\begin{aligned}
\nu\left[\mathbf{q}_{j+1}, \mathbf{p}_{0} ; t_{j+1}, t_{0}\right]= & \nu\left[\mathbf{q}_{j+1}, \mathbf{p}_{j} ; t_{j+1}, t_{j}\right]+\nu\left[\mathbf{q}_{j}, \mathbf{p}_{0} ; t_{j}, t_{0}\right] \\
& +\frac{1}{2}\left(\mu_{j}-\widetilde{\sigma}_{j}\right)
\end{aligned}
$$

for adding up this type of index for any two finite joining trajectory segments. For the case of a sufficiently small time interval $\left[t_{j}, t_{j+1}\right]$ this reduces to the infinitesimal additive property [cf. Eq. (26)]

$$
\nu\left[\mathbf{q}_{j+1}, \mathbf{p}_{0} ; t_{j+1}, t_{0}\right]=\nu\left[\mathbf{q}_{j}, \mathbf{p}_{0} ; t_{j}, t_{0}\right]+\frac{1}{2}\left(\mu_{j}-\widetilde{\sigma}_{j}\right) .
$$

The integer $\mu_{j}$ is given by Eq. (27) and $\widetilde{\sigma_{j}}$ by [cf. Eq. (28)]

$$
\begin{aligned}
\sigma_{j}^{\sim} & =\operatorname{sig}\left\{\left[\partial_{\mathbf{p}_{j}} \mathbf{q}\left(\mathbf{q}_{j}, \mathbf{p}_{j} ; t_{j+1}, t_{j}\right)\right]^{-1} \partial_{\mathbf{q}_{0}} \mathbf{q}\left(t_{j+1}\right)\left[\partial_{\mathbf{q}_{0}} \mathbf{q}\left(t_{j}\right)\right]^{-1}\right\} \\
& =\operatorname{sig}\left\{\partial_{\mathbf{q}_{0}} \mathbf{q}\left(t_{j}\right)\left[\partial_{\mathbf{q}_{0}} \mathbf{q}\left(t_{j+1}\right)\right]^{-1} \partial_{\mathbf{p}_{j}} \mathbf{q}\left(\mathbf{q}_{j}, \mathbf{p}_{j} ; t_{j+1}, t_{j}\right)\right\} \\
& =\operatorname{sig}\left\{\mathbf{B}\left(t_{j+1}, t_{j}\right)^{-1} \mathbf{A}\left(t_{j+1}\right) \mathbf{A}\left(t_{j}\right)^{-1}\right\} \\
& =\operatorname{sig}\left\{\mathbf{A}\left(t_{j}\right) \mathbf{A}\left(t_{j+1}\right)^{-1} \mathbf{B}\left(t_{j+1}, t_{j}\right)\right\}
\end{aligned}
$$

The index for a global interval $\left[t_{0}, t\right]$ is then given by

$$
\nu\left[\mathbf{q}, \mathbf{p}_{0} ; t, t_{0}\right]=\frac{1}{2} \sum_{j}\left(\mu_{j}-\widetilde{\sigma}_{j}\right),
$$

with the initial index being zero.

For the final momentum representations the roles of the canonical coordinates and momenta are reversed. Within the above equations for the final coordinate representations, this switches all stability matrices $\mathbf{A}, \mathbf{B} \Rightarrow \mathbf{D},-\mathbf{C}$, respectively, and the classical action generators $\phi, \widetilde{\phi} \Rightarrow \Phi, \widetilde{\Phi}$ defined in Appendix B. Hence the analogous semiclassical matrix elements take their required (nonuniform) semiclassical forms

$$
\begin{aligned}
\left\langle\mathbf{p}\left|\hat{U}\left(t, t_{0}\right)\right| \mathbf{q}_{0}\right\rangle^{\mathrm{sc}}= & (-2 \pi i \hbar)^{-N / 2}\left|\operatorname{det} \partial_{\mathbf{p}_{0}} \mathbf{p}(t)\right|^{-1 / 2} \\
& \times \exp \left[\frac{i}{\hbar} \widetilde{\Phi}\left(\mathbf{p}, \mathbf{q}_{0} ; t, t_{0}\right)-i \frac{\pi}{2} \nu\left[\mathbf{p}, \mathbf{q}_{0} ; t, t_{0}\right]\right],
\end{aligned}
$$

$$
\begin{aligned}
\left\langle\mathbf{p}\left|\hat{U}\left(t, t_{0}\right)\right| \mathbf{p}_{0}\right\rangle^{\mathrm{sc}}= & (2 \pi i \hbar)^{-N / 2}\left|\operatorname{det} \partial_{\mathbf{q}_{0}} \mathbf{p}(t)\right|^{-1 / 2} \\
& \times \exp \left[\frac{i}{\hbar} \Phi\left(\mathbf{p}, \mathbf{p}_{0} ; t, t_{0}\right)-i \frac{\pi}{2} \nu\left[\mathbf{p}, \mathbf{p}_{0} ; t, t_{0}\right]\right] .
\end{aligned}
$$


The properties for adding the index for any two joining finite trajectory segments, analogous to Eqs. (34) and (37), are then

$$
\begin{aligned}
\nu\left[\mathbf{p}_{j+1}, \mathbf{p}_{0} ; t_{j+1}, t_{0}\right]= & \nu\left[\mathbf{p}_{j+1}, \mathbf{p}_{j} ; t_{j+1}, t_{j}\right]+\nu\left[\mathbf{p}_{j}, \mathbf{p}_{0} ; t_{j}, t_{0}\right] \\
& +\frac{1}{2}\left(N-\bar{\sigma}_{j}\right)
\end{aligned}
$$

and

$$
\begin{aligned}
\nu\left[\mathbf{p}_{j+1}, \mathbf{q}_{0} ; t_{j+1}, t_{0}\right]= & \nu\left[\mathbf{p}_{j+1}, \mathbf{q}_{j} ; t_{j+1}, t_{j}\right]+\nu\left[\mathbf{p}_{j}, \mathbf{q}_{0} ; t_{j}, t_{0}\right] \\
& +\frac{1}{2}\left(\bar{\mu}_{j}-\overline{\widetilde{\sigma}}_{j}\right),
\end{aligned}
$$

where

$$
\begin{aligned}
\overline{\widetilde{\sigma}}_{j} & =-\operatorname{sig}\left\{\left[\partial_{\mathbf{q}_{j}} \mathbf{p}\left(\mathbf{q}_{j}, \mathbf{p}_{j} ; t_{j+1}, t_{j}\right)\right]^{-1} \partial_{\mathbf{p}_{0}} \mathbf{p}\left(t_{j+1}\right)\left[\partial_{\mathbf{p}_{0}} \mathbf{p}\left(t_{j}\right)\right]^{-1}\right\} \\
& =-\operatorname{sig}\left\{\partial_{\mathbf{p}_{0}} \mathbf{p}\left(t_{j}\right)\left[\partial_{\mathbf{p}_{0}} \mathbf{p}\left(t_{j+1}\right)\right]^{-1} \partial_{\mathbf{q}_{j}} \mathbf{p}\left(\mathbf{q}_{j}, \mathbf{p}_{j} ; t_{j+1}, t_{j}\right)\right\} \\
& =-\operatorname{sig}\left\{\mathbf{C}\left(t_{j+1}, t_{j}\right)^{-1} \mathbf{D}\left(t_{j+1}\right) \mathbf{D}\left(t_{j}\right)^{-1}\right\} \\
& =-\operatorname{sig}\left\{\mathbf{D}\left(t_{j}\right) \mathbf{D}\left(t_{j+1}\right)^{-1} \mathbf{C}\left(t_{j+1}, t_{j}\right)\right\}, \\
\bar{\sigma}_{j} & =-\operatorname{sig}\left\{\left[\partial_{\mathbf{q}_{j}} \mathbf{p}\left(\mathbf{q}_{j}, \mathbf{p}_{j} ; t_{j+1}, t_{j}\right)\right]^{-1} \partial_{\mathbf{q}_{0}} \mathbf{p}\left(t_{j+1}\right)\left[\partial_{\mathbf{q}_{0}} \mathbf{p}\left(t_{j}\right)\right]^{-1}\right\} \\
& =-\operatorname{sig}\left\{\partial_{\mathbf{q}_{0}} \mathbf{p}\left(t_{j}\right)\left[\partial_{\mathbf{q}_{0}} \mathbf{p}\left(t_{j+1}\right)\right]^{-1} \partial_{\mathbf{q}_{j}} \mathbf{p}\left(\mathbf{q}_{j}, \mathbf{p}_{j} ; t_{j+1}, t_{j}\right)\right\} \\
& =-\operatorname{sig}\left\{\mathbf{C}\left(t_{j+1}, t_{j}\right)^{-1} \mathbf{C}\left(t_{j+1}\right) \mathbf{C}\left(t_{j}\right)^{-1}\right\} \\
& =-\operatorname{sig}\left\{\mathbf{C}\left(t_{j}\right) \mathbf{C}\left(t_{j+1}\right)^{-1} \mathbf{C}\left(t_{j+1}, t_{j}\right)\right\}, \\
\bar{\mu}_{j} & =-\operatorname{sig}\left\{\left[\partial_{\mathbf{p}_{j}} \mathbf{p}\left(\mathbf{q}_{j}, \mathbf{p}_{j} ; t_{j+1}, t_{j}\right)\right]^{-1} \partial_{\mathbf{q}_{j}} \mathbf{p}\left(\mathbf{q}_{j}, \mathbf{p}_{j} ; t_{j+1}, t_{j}\right)\right\} \\
& =-\operatorname{sig}\left\{\partial_{\mathbf{q}_{j}} \mathbf{p}\left(\mathbf{q}_{j}, \mathbf{p}_{j} ; t_{j+1}, t_{j}\right)^{-1} \partial_{\mathbf{p}_{j}} \mathbf{p}\left(\mathbf{q}_{j}, \mathbf{p}_{j} ; t_{j+1}, t_{j}\right)\right\} \\
& \equiv-\operatorname{sig} \mathbf{D}\left(t_{j+1}, t_{j}\right)^{-1} \mathbf{C}\left(t_{j+1}, t_{j}\right) \\
& =-\operatorname{sig} \mathbf{C}\left(t_{j+1}, t_{j}\right)^{-1} \mathbf{D}\left(t_{j+1}, t_{j}\right) .
\end{aligned}
$$

Within the small $t_{j+1}-t_{j}$ limit we also have $\bar{\mu}_{j} \rightarrow-\operatorname{sig} \mathbf{C}\left(t_{j+1}, t_{j}\right)$. Equations (43) and (44) also give rise to the infinitesimal index addition properties [cf. Eqs. (26) and (38)]

$$
\nu\left[\mathbf{p}_{j+1}, \mathbf{p}_{0} ; t_{j+1}, t_{0}\right]=\nu\left[\mathbf{p}_{j}, \mathbf{p}_{0} ; t_{j}, t_{0}\right]+\frac{1}{2}\left(\bar{\mu}_{j}-\bar{\sigma}_{j}\right)
$$

and

$$
\nu\left[\mathbf{p}_{j+1}, \mathbf{q}_{0} ; t_{j+1}, t_{0}\right]=\nu\left[\mathbf{p}_{j}, \mathbf{q}_{0} ; t_{j}, t_{0}\right]+\frac{1}{2}\left(\bar{\mu}_{j}-\overline{\widetilde{\sigma}}_{j}\right),
$$

with the initial index formula [cf. Eq. (17)]

$$
\begin{aligned}
\nu\left[\mathbf{p}\left(t_{0}^{+}\right), \mathbf{p}_{0} ; t_{0}^{+}, t_{0}\right] & =-\frac{1}{2}\left[\operatorname{sig}\left\{\left[\partial_{\mathbf{p}_{0}} \mathbf{p}\left(t_{0}^{+}\right)\right]^{-1} \partial_{\mathbf{q}_{0}} \mathbf{p}\left(t_{0}^{+}\right)\right\}+N\right] \\
& =-\frac{1}{2}\left[\operatorname{sig} \mathbf{D}\left(t_{0}^{+}\right)^{-1} \mathbf{C}\left(t_{0}^{+}\right)+N\right] \\
& =-\frac{1}{2}\left[\operatorname{sig} \partial_{\mathbf{q}_{0}} \mathbf{p}\left(t_{0}^{+}\right)+N\right]
\end{aligned}
$$

The initial index in Eq. (41) is zero, i.e., $\nu\left[\mathbf{p}\left(t_{0}^{+}\right), \mathbf{q}_{0} ; t_{0}^{+}, t_{0}\right]=0$. Finally, the indices for a global interval $\left[t_{0}, t\right]$ are given by [cf. Eqs. (29) and (40)]

$$
\begin{aligned}
\nu\left[\mathbf{p}, \mathbf{p}_{0} ; t, t_{0}\right]= & \nu\left[\mathbf{p}\left(t_{0}^{+}\right), \mathbf{p}_{0} ; t_{0}^{+}, t_{0}\right]+\frac{1}{2} \sum_{j}\left(\bar{\mu}_{j}-\bar{\sigma}_{j}\right), \\
& \nu\left[\mathbf{p}, \mathbf{q}_{0} ; t, t_{0}\right]=\frac{1}{2} \sum_{j}\left(\bar{\mu}_{j}-\overline{\widetilde{\sigma}}_{j}\right) .
\end{aligned}
$$

Note, finally, that the relevant indices for any other possible representations, such as partially mixed coordinate and momentum representations of the propagator, may also be derived as obvious extensions of this treatment.

\section{B. Index connecting relations}

In this section we show how the SSP process gives rise to "connecting" relations that relate the indices in any one representation to that in any other representation. For simplicity we denote the four basic types of indices by $\nu_{1}=\nu\left[\mathbf{q}, \mathbf{q}_{0} ; t, t_{0}\right], \nu_{2}=\nu\left[\mathbf{q}, \mathbf{p}_{0} ; t, t_{0}\right], \nu_{3}=\nu\left[\mathbf{p}, \mathbf{q}_{0} ; t, t_{0}\right]$, and $\nu_{4}=\nu\left[\mathbf{p}, \mathbf{p}_{0} ; t, t_{0}\right]$. Consider first the relationship between $\nu_{1}$ and $\nu_{2}$. In accord with the preceding subsection, it is clear that we can proceed by considering the relation

$$
\begin{aligned}
\left\langle\mathbf{q}\left|U\left(t, t_{0}\right)\right| \mathbf{p}_{0}\right\rangle^{\mathrm{sc}} & \sim \int d \mathbf{q}_{0}\left\langle\mathbf{q}\left|\hat{U}\left(t, t_{0}\right)\right| \mathbf{q}_{0}\right\rangle^{\mathrm{sc}}\left\langle\mathbf{q}_{0} \mid \mathbf{p}_{0}\right\rangle \\
\sim & (2 \pi i \hbar)^{-N} \int d \mathbf{q}_{0}\left|\operatorname{det} \partial_{\mathbf{p}_{0}} \mathbf{q}(t)\right|^{-1 / 2} \\
& \times \exp \left[\frac{i}{\hbar}\left[\phi\left(\mathbf{q}, \mathbf{q}_{0} ; t, t_{0}\right)+\mathbf{q}_{0} \cdot \mathbf{p}_{0}\right]-i \frac{\pi}{2} \nu_{1}\right]
\end{aligned}
$$

within the stationary-phase approximation.

The stationary-phase condition is $\mathbf{p}_{0}$ $=-\partial_{\mathbf{q}_{0}} \phi\left(\mathbf{q}, \mathbf{q}_{0} ; t, t_{0}\right)$, which shows that, according to the first set of generator relations in Eq. (B2), all the stationaryphase points $\mathbf{q}_{0}=\mathbf{q}_{0}^{(k)}$ correspond to the classical root trajectories starting with $\mathbf{p}_{0}$ and ending with $\mathbf{q}=\mathbf{q}\left(\mathbf{q}_{0}, \mathbf{p}_{0} ; t, t_{0}\right)$. These trajectories are, as required, the same as those within Eq. (35). Using the second of Eqs. (B3), the Hessian of the above integral phase is $\partial_{\mathbf{q}_{0}}^{2} \phi\left(\mathbf{q}, \mathbf{q}_{0} ; t, t_{0}\right)$ $=\left[\partial_{\mathbf{p}_{0}} \mathbf{q}(t)\right]^{-1} \cdot \partial_{\mathbf{q}_{0}} \mathbf{q}(t)$. Taking the determinant and signature of this Hessian and substituting these into the stationaryphase formula for the integral in Eq. (53) then recovers the semiclassical result of Eq. (35). In addition it establishes an index connecting relation between $\nu_{2}$ and $\nu_{1}$,

$$
\begin{aligned}
\nu_{2} & =\nu_{1}+\frac{1}{2}\left(N-\operatorname{sig}\left\{\left[\partial_{\mathbf{p}_{0}} \mathbf{q}(t)\right]^{-1} \cdot \partial_{\mathbf{q}_{0}} \mathbf{q}(t)\right\}\right) \\
& =\nu_{1}+\frac{1}{2}\left[N-\operatorname{sig} \mathbf{B}^{-1} \mathbf{A}\right]=\nu_{1}+\frac{1}{2}\left[N-\operatorname{sig} \mathbf{A} \mathbf{B}^{T}\right] .
\end{aligned}
$$

Here for the stability matrices we have used the notation of Appendix C. The last expression follows from Eq. (C9). Equation (54) is clearly just a consequence of applying a different ordering of the SSP process than was used to obtain Eq. (35). It is understood that in these connecting relations all matrices appearing in the equations are assumed to be nonsingular at time $t$, in accord with the SSP discussion above.

It is important to emphasize the content of an index connecting relation such as Eq. (54). For any classical trajectory 
it gives the value of a particular index in terms of another index that can be assigned to the same trajectory for time $t$. For instance, $\nu_{1}$ can be computed using Eq. (29) and $\nu_{2}$ is given analogously by Eq. (40). Those expressions give particular ways of computing the global value of the separate indices and their respective changes. In fact, from these formulas changes in either of the indices are readily seen to be due to the crossing of the respective caustics. Equation (54), however, provides a direct connection between $\nu_{1}$ and $\nu_{2}$ so that once one is computed the other follows by simply adding an end-point signature term at final time $t$. Moreover, the connecting formulas then also provide us with straightforward alternatives in which to obtain a particular type of index change in terms of another along the same trajectory segment. For instance, Eq. (54) can be used to give

$$
\begin{aligned}
\Delta \nu_{2}\left[t \rightarrow t^{\prime}\right]= & \Delta \nu_{1}\left[t \rightarrow t^{\prime}\right]+\frac{1}{2}\left[\operatorname{sig} \mathbf{A}(t) \mathbf{B}(t)^{T}\right. \\
& \left.-\operatorname{sig} \mathbf{A}\left(t^{\prime}\right) \mathbf{B}\left(t^{\prime}\right)^{T}\right]
\end{aligned}
$$

for a time segment $\left[t, t^{\prime}\right]$. The SSP procedure can be continued to relate any other of the representations, exhausting any number of index connecting relations desired. Here we simply provide some of useful connection identities among the four basic types of indices. A stationary-phase transformation from $\mathbf{q}$ to $\mathbf{p}$ on Eq. (53), for example, gives rise to a relation between $\nu_{1}$ and $\nu_{4}$,

$$
\nu_{4}=\nu_{1}-\frac{1}{2}\left[\operatorname{sig} \mathbf{B}^{-1} \mathbf{A}+\operatorname{sig} \mathbf{A} \mathbf{C}^{-1}\right] .
$$

Similar SSP applications give rise to other connecting relations such as

$$
\begin{aligned}
& \nu_{3}=\nu_{1}+\frac{1}{2}\left[N-\operatorname{sig} \mathbf{D B} \mathbf{B}^{-1}\right], \\
& \nu_{4}=\nu_{3}-\frac{1}{2}\left[N+\operatorname{sig} \mathbf{D}^{-1} \mathbf{C}\right] .
\end{aligned}
$$

We can of course also combine any pair of the above relations to give further results. Moreover, it is clear that one can also generate several forms of such relations for the same pair of indices based on different SSP routes. For instance, the combination of Eqs. (56) and (57) leads to a relation between $\nu_{1}$ and $\nu_{4}$ that is alternate to Eq. (55). Extensions of the connecting relations also follow similarly for indices of any other mixed coordinate and momentum representations and these can also be used to further describe index changes along a trajectory. These connecting relations are some of the essential aspects that arise in Maslov's theory [4,14]. We have therefore shown that the applications of SSP operations, with assorted orderings, produce various index formulas that are really properties of any classical trajectory or symplectic evolution.

\section{CONCLUSION}

We have shown, based on straightforward sequential stationary-phase procedures, that differing sequences of stationary-phase operations among the nonuniform propagator matrix elements in the various coordinate and momentum representations lead to various useful index formulas and general index properties. All index formulas presented here are easily implemented using classical trajectories.

This work provides an alternative, and much simplified, approach to Maslov index theory [4] in which we have derived a canonical structure of semiclassical phase indices for the propagator based solely on the simple application of sequential stationary phase and the use of a generally asymptotic path-integral approach. The present theory therefore gives the nonuniform semiclassical structure for matrix elements of unitary transformations induced by the family of propagatorlike operators. Of future interest is related work on the phase indices associated with the canonical structure of the nonuniform semiclassical matrix elements for more general classes of unitary transformations.

\section{APPENDIX A: CONDITIONS FOR SEQUENTIAL STATIONARY PHASE}

Properties (i)-(iii) [see Eq. (7)] are essential for the replacement of the full stationary-phase result by sequential stationary phase. Here we prove these results, given Eqs. (4)-(6).

From an implicit function theorem we can differentiate $\widetilde{\varphi}$ with respect to $\mathbf{x}$ giving

$$
\partial_{\mathbf{x}} \widetilde{\varphi}(\mathbf{x})=\partial_{\mathbf{x}} \varphi(\mathbf{x}, \widetilde{\mathbf{y}}(\mathbf{x}))+\partial_{\mathbf{y}} \varphi(\mathbf{x}, \widetilde{\mathbf{y}}(\mathbf{x})) \cdot \partial_{\mathbf{x}} \tilde{\mathbf{y}}(\mathbf{x})
$$

Equation (4) gives $\partial_{\mathbf{y}} \varphi(\mathbf{x}, \widetilde{\mathbf{y}}(\mathbf{x}))=\mathbf{0}$. Using this and Eq. (6) in Eq. (A1) also gives $\partial_{\mathbf{x}} \varphi\left(\mathbf{x}^{*}, \widetilde{\mathbf{y}}\left(\mathbf{x}^{*}\right)\right)=\mathbf{0}$, hence proving property (i). Another differentiation of Eq. (A1) gives

$$
\partial_{\mathbf{x}}^{2} \widetilde{\varphi}=\partial_{\mathbf{x}}^{2} \varphi(\mathbf{x}, \widetilde{\mathbf{y}}(\mathbf{x}))+\partial_{\mathbf{y}} \partial_{\mathbf{x}} \varphi(\mathbf{x}, \widetilde{\mathbf{y}}(\mathbf{x})) \cdot \partial_{\mathbf{x}} \widetilde{\mathbf{y}}(\mathbf{x})
$$

and using the identity obtained by implicitly differentiating Eq. (4)

$$
\partial_{\mathbf{y}}^{2} \varphi(\mathbf{x}, \widetilde{\mathbf{y}}(\mathbf{x})) \cdot \partial_{\mathbf{x}} \widetilde{\mathbf{y}}(\mathbf{x})+\partial_{\mathbf{x}} \partial_{\mathbf{y}} \varphi(\mathbf{x}, \widetilde{\mathbf{y}}(\mathbf{x}))=\mathbf{0}
$$

gives the Hessian identity

$$
\begin{aligned}
\partial_{\mathbf{x}}^{2} \widetilde{\varphi}= & \partial_{\mathbf{x}}^{2} \varphi(\mathbf{x}, \widetilde{\mathbf{y}}(\mathbf{x})) \\
& -\partial_{\mathbf{y}} \partial_{\mathbf{x}} \varphi(\mathbf{x}, \widetilde{\mathbf{y}}(\mathbf{x})) \cdot\left[\partial_{\mathbf{y}}^{2} \varphi(\mathbf{x}, \widetilde{\mathbf{y}}(\mathbf{x}))\right]^{-1} \cdot\left[\partial_{\mathbf{y}} \partial_{\mathbf{x}} \varphi(\mathbf{x}, \widetilde{\mathbf{y}}(\mathbf{x}))\right]^{T}
\end{aligned}
$$

This involves the matrix inverse of the sub-Hessian $\partial_{\mathbf{y}}^{2} \varphi$, which exists because of Eq. (5); the superscript $T$ denotes the transpose. Of course, Eq. (A2) is true for $\mathbf{x}=\mathbf{x}_{*}$. To show (ii) we must show

$$
\begin{aligned}
\operatorname{det} \partial_{(\mathbf{x}, \mathbf{y})}^{2} \varphi(\mathbf{x}, \widetilde{\mathbf{y}}(\mathbf{x})) & \left.\equiv \operatorname{det}\left(\begin{array}{cc}
\partial_{\mathbf{x}}^{2} \varphi & \partial_{\mathbf{y}} \partial_{\mathbf{x}} \varphi \\
{\left[\partial_{\mathbf{y}} \partial_{\mathbf{x}} \varphi\right]^{T}} & \partial_{\mathbf{y}}^{2} \varphi
\end{array}\right)\right|_{\mathbf{y}=\widetilde{\mathbf{y}}(\mathbf{x})} \\
& =\operatorname{det} \partial_{\mathbf{y}}^{2} \varphi(\mathbf{x}, \widetilde{\mathbf{y}}(\mathbf{x})) \operatorname{det} \partial_{\mathbf{x}}^{2} \widetilde{\varphi}
\end{aligned}
$$

This equality follows via the useful matrix identity [8] 


$$
\begin{aligned}
& \left(\begin{array}{cc}
\mathbf{I}_{m} & \mathbf{0}_{m \times n} \\
-\mathbf{D}^{-1} \mathbf{C}^{T} & \mathbf{I}_{n}
\end{array}\right)^{T}\left(\begin{array}{cc}
\mathbf{B} & \mathbf{C} \\
\mathbf{C}^{T} & \mathbf{D}
\end{array}\right)\left(\begin{array}{cc}
\mathbf{I}_{m} & \mathbf{0}_{m \times n} \\
-\mathbf{D}^{-1} \mathbf{C}^{T} & \mathbf{I}_{n}
\end{array}\right) \\
& =\left(\begin{array}{cc}
\mathbf{B}-\mathbf{C D}^{-1} \mathbf{C}^{T} & \mathbf{0}_{m \times n} \\
\mathbf{0}_{n \times m} & \mathbf{D}
\end{array}\right),
\end{aligned}
$$

where $\mathbf{B}, \mathbf{C}$, and $\mathbf{D}$ are any $m \times m, m \times n$, and $n \times n$ matrices, respectively, and $\mathbf{D}$ is symmetric nonsingular; $\mathbf{I}_{m}$ is the identity $m \times m$ matrix and $\mathbf{0}_{m \times n}$ is the $m \times n$ matrix of zeros. Taking determinants of Eq. (A4) and using the fact that the $(m+n) \times(m+n)$ matrix (and its transpose) involving submatrices $\mathbf{I}_{m}, \mathbf{I}_{n}$ has a unit determinant gives

$$
\operatorname{det}\left(\begin{array}{cc}
\mathbf{B} & \mathbf{C} \\
\mathbf{C}^{T} & \mathbf{D}
\end{array}\right)=\operatorname{det} \mathbf{D} \operatorname{det}\left(\mathbf{B}-\mathbf{C D}^{-1} \mathbf{C}^{T}\right)
$$

Now letting $\mathbf{B}=\partial_{\mathbf{x}}^{2} \varphi, \mathbf{C}=\partial_{\mathbf{y}} \partial_{\mathbf{x}} \varphi$, and $\mathbf{D}=\partial_{\mathbf{y}}^{2} \varphi$ in Eq. (A5) gives Eq. (A3) and hence proves property (ii). Property (iii) follows directly from Eq. (A4), and by the property $\operatorname{sig} \mathbf{P}^{T} \mathbf{M P}=\operatorname{sig} \mathbf{M}$ for any invertible matrix $\mathbf{P}$ inducing such a congruence transformation. The latter identity is seen by applying the Morse lemma [6] to nondegenerate quadratic forms $f, f^{\prime}$ defined by $f(\mathbf{z}) \equiv \Sigma_{i j}\left(\mathbf{P}^{T} \mathbf{M P}\right)_{i j} z_{i} z_{j}$ $=\Sigma_{i j} \mathbf{M}_{i j} z_{i}^{\prime} z_{j}^{\prime} \equiv f^{\prime}\left(\mathbf{z}^{\prime}\right)$, where $z_{i}^{\prime}=\Sigma_{k} P_{i k} z_{k}$. Morse's lemma then immediately gives $\operatorname{sig} \partial_{\mathbf{z}}^{2} f(\mathbf{0})=\operatorname{sig} \partial_{\mathbf{z}^{\prime}}^{2} f^{\prime}(\mathbf{0})$, and hence $\operatorname{sig} \mathbf{P}^{T} \mathbf{M P}=\operatorname{sig} \mathbf{M}$. Making the obvious identification of $\mathbf{P}$ as the third $(m+n) \times(m+n)$ matrix appearing on the left-hand side of Eq. (A4), then proves Eq. (8) and completes the proof.

\section{APPENDIX B: CLASSICAL GENERATOR RELATIONS}

In this appendix we present some basic useful identities for the four types of classical generators used in the theory of Sec. III. The first set of properties concerns the classical action expressed as a function of initial and final coordinates and time variables as given by

$$
\phi\left(\mathbf{q}, \mathbf{q}_{0} ; t, t_{0}\right)=\int_{t_{0}}^{t} d t^{\prime}\left[\mathbf{p}\left(t^{\prime}\right) \cdot \dot{\mathbf{q}}\left(t^{\prime}\right)-H\left(\mathbf{q}\left(t^{\prime}\right), \mathbf{p}\left(t^{\prime}\right), t^{\prime}\right)\right]
$$

where the initial momentum $\mathbf{p}_{0}$ within the trajectory functions $\mathbf{q}(t)=\mathbf{q}\left(\mathbf{q}_{0}, \mathbf{p}_{0} ; t, t_{0}\right)$ and $\mathbf{p}(t)=\mathbf{p}\left(\mathbf{q}_{0}, \mathbf{p}_{0} ; t, t_{0}\right)$ is considered as a function of the variables $\mathbf{q}, \mathbf{q}_{0}, t, t_{0}$ implicitly via the trajectory root equation $\mathbf{q}(t)=\mathbf{q}$.

Every such action integral satisfies the useful generator relations [12]

$$
\mathbf{p}_{0}=-\partial_{\mathbf{q}_{0}} \phi\left(\mathbf{q}, \mathbf{q}_{0} ; t, t_{0}\right), \quad \mathbf{p}(t)=\partial_{\mathbf{q}} \phi\left(\mathbf{q}, \mathbf{q}_{0} ; t, t_{0}\right) .
$$

Differentiating these relations gives rise to other useful identities for expressing various Hessian matrices of the generator in terms of stability matrices for any given trajectory:

$$
\begin{gathered}
-\partial_{\mathbf{q}} \partial_{\mathbf{q}_{0}} \phi \cdot \partial_{\mathbf{p}_{0}} \mathbf{q}(t)=\mathbf{I}_{N}, \quad \partial_{\mathbf{q}_{0}}^{2} \phi=\left[\partial_{\mathbf{p}_{0}} \mathbf{q}(t)\right]^{-1} \cdot \partial_{\mathbf{q}_{0}} \mathbf{q}(t), \\
\partial_{\mathbf{q}}^{2} \phi=\partial_{\mathbf{p}_{0}} \mathbf{p}(t) \cdot\left[\partial_{\mathbf{p}_{0}} \mathbf{q}(t)\right]^{-1} \\
\partial_{\mathbf{q}}^{2} \phi \cdot \partial_{\mathbf{q}_{0}} \mathbf{q}(t)+\left[\partial_{\mathbf{q}} \partial_{\mathbf{q}_{0}} \phi\right]^{T}=\partial_{\mathbf{q}_{0}} \mathbf{p}(t)
\end{gathered}
$$

Other classical generators considered explicitly as functions of other combinations of initial and final canonical variables are just Legendre transforms of one another. A generator of motion expressed as a function of the initial momentum and final coordinate is given by

$$
\widetilde{\phi}\left(\mathbf{q}, \mathbf{p}_{0} ; t, t_{0}\right)=\phi\left(\mathbf{q}, \mathbf{q}_{0} ; t, t_{0}\right)+\mathbf{q}_{0} \cdot \mathbf{p}_{0},
$$

where $\mathbf{q}_{0}$ is here considered as a function of $\mathbf{q}$ and $\mathbf{p}_{0}$, and the times, by inverting the trajectory root equation or by inverting the first relation in Eqs. (B2). Differentiating Eq. (B4) while using Eqs. (B2) gives yet another set of generator relations

$$
\mathbf{q}_{0}=\partial_{\mathbf{p}_{0}} \widetilde{\phi}\left(\mathbf{q}, \mathbf{p}_{0} ; t, t_{0}\right), \quad \mathbf{p}(t)=\partial_{\mathbf{q}} \widetilde{\phi}\left(\mathbf{q}, \mathbf{p}_{0} ; t, t_{0}\right) .
$$

The analogs of Eqs. (B3) are obtained by differentiating Eqs. (B5);

$$
\begin{gathered}
\partial_{\mathbf{q}} \partial_{\mathbf{p}_{0}} \widetilde{\phi} \cdot \partial_{\mathbf{q}_{0}} \mathbf{q}(t)=\mathbf{I}_{N}, \quad \partial_{\mathbf{p}_{0}}^{2} \widetilde{\phi}=-\left[\partial_{\mathbf{q}_{0}} \mathbf{q}(t)\right]^{-1} \cdot \partial_{\mathbf{p}_{0}} \mathbf{q}(t), \\
\partial_{\mathbf{q}}^{2} \widetilde{\phi}=\partial_{\mathbf{q}_{0}} \mathbf{p}(t) \cdot\left[\partial_{\mathbf{q}_{0}} \mathbf{q}(t)\right]^{-1} \\
\partial_{\mathbf{q}}^{2} \widetilde{\phi} \cdot \partial_{\mathbf{p}_{0}} \mathbf{q}(t)+\left[\partial_{\mathbf{q}} \partial_{\mathbf{p}_{0}} \widetilde{\phi}\right]^{T}=\partial_{\mathbf{p}_{0}} \mathbf{p}(t) .
\end{gathered}
$$

Two other useful basic forms of the generators are those explicitly involving the final momenta. One such type of generator of motion is a function of the initial coordinate and final momenta and can be defined via $\phi$,

$$
\widetilde{\Phi}\left(\mathbf{p}, \mathbf{q}_{0} ; t, t_{0}\right)=\phi\left(\mathbf{q}, \mathbf{q}_{0} ; t, t_{0}\right)-\mathbf{q} \cdot \mathbf{p}
$$

with $\mathbf{q}$ considered as a function of $\mathbf{q}_{0}$ and $\mathbf{p}$, and the times, by inverting the trajectory root equation $\mathbf{p}(t)=\mathbf{p}$ or by inverting the second relation in Eqs. (B2). Differentiating Eq. (B7) while again using Eqs. (B2) gives the set of generator relations

$$
\mathbf{p}_{0}=-\partial_{\mathbf{q}_{0}} \widetilde{\Phi}\left(\mathbf{p}, \mathbf{q}_{0} ; t, t_{0}\right), \quad \mathbf{q}(t)=-\partial_{\mathbf{p}} \widetilde{\Phi}\left(\mathbf{p}, \mathbf{q}_{0} ; t, t_{0}\right) .
$$

Using these relations in the same manner as above gives the analogs of Eqs. (B3) or (B6),

$$
\begin{gathered}
-\partial_{\mathbf{p}} \partial_{\mathbf{q}_{0}} \widetilde{\Phi} \cdot \partial_{\mathbf{p}_{0}} \mathbf{p}(t)=\mathbf{I}_{N}, \quad \partial_{\mathbf{q}_{0}}^{2} \widetilde{\Phi}=\left[\partial_{\mathbf{p}_{0}} \mathbf{p}(t)\right]^{-1} \cdot \partial_{\mathbf{q}_{0}} \mathbf{p}(t), \\
\partial_{\mathbf{p}}^{2} \widetilde{\Phi}=-\partial_{\mathbf{p}_{0}} \mathbf{q}(t) \cdot\left[\partial_{\mathbf{p}_{0}} \mathbf{p}(t)\right]^{-1}, \\
\partial_{\mathbf{p}}^{2} \widetilde{\Phi} \cdot \partial_{\mathbf{q}_{0}} \mathbf{p}(t)+\left[\partial_{\mathbf{p}} \partial_{\mathbf{q}_{0}} \widetilde{\phi}\right]^{T}=-\partial_{\mathbf{q}_{0}} \mathbf{q}(t) .
\end{gathered}
$$

The last type of generator considered is a function of the initial and final momenta and again can be defined via the other generators. In terms of $\widetilde{\phi}$, for instance, it is given by

$$
\Phi\left(\mathbf{p}, \mathbf{p}_{0} ; t, t_{0}\right)=\widetilde{\phi}\left(\mathbf{q}, \mathbf{p}_{0} ; t, t_{0}\right)-\mathbf{q} \cdot \mathbf{p}
$$


with $\mathbf{q}$ here considered as function of $\mathbf{p}_{0}$ and $\mathbf{p}$, and the times, and obtained by inverting the second relation in Eqs. (B5). Differentiating Eq. (B10) while using Eqs. (B5) gives the set of generator relations

$\mathbf{q}_{0}=\partial_{\mathbf{p}_{0}} \Phi\left(\mathbf{p}, \mathbf{p}_{0} ; t, t_{0}\right), \quad \mathbf{q}(t)=-\partial_{\mathbf{p}} \Phi\left(\mathbf{p}, \mathbf{p}_{0} ; t, t_{0}\right)$

Analogous relations to the above then follow using Eqs. (B11),

$$
\begin{gathered}
\partial_{\mathbf{p}} \partial_{\mathbf{p}_{0}} \Phi \cdot \partial_{\mathbf{q}_{0}} \mathbf{p}(t)=\mathbf{I}_{N}, \quad \partial_{\mathbf{p}_{0}}^{2} \Phi=-\left[\partial_{\mathbf{q}_{0}} \mathbf{p}(t)\right]^{-1} \cdot \partial_{\mathbf{p}_{0}} \mathbf{p}(t), \\
\partial_{\mathbf{p}}^{2} \Phi=-\partial_{\mathbf{q}_{0}} \mathbf{q}(t) \cdot\left[\partial_{\mathbf{q}_{0}} \mathbf{p}(t)\right]^{-1} \\
\partial_{\mathbf{p}}^{2} \Phi \cdot \partial_{\mathbf{p}_{0}} \mathbf{p}(t)+\left[\partial_{\mathbf{p}} \partial_{\mathbf{p}_{0}} \Phi\right]^{T}=-\partial_{\mathbf{p}_{0}} \mathbf{q}(t)
\end{gathered}
$$

It is instructive to note that, for instance, the identities of Eqs. (B9) and (B12) are consistently obtained by making a global canonical change of variables $\mathbf{q} \rightarrow-\mathbf{p}, \mathbf{p} \rightarrow \mathbf{q}$, which basically reverses the roles of the coordinates and momenta for all $t$, with $\phi \rightarrow \Phi$ and $\widetilde{\phi} \rightarrow \widetilde{\Phi}$ within Eqs. (B3) and (B6). Note that the above are just the properties of the $F_{i}$-type generating functions [12].

\section{APPENDIX C: STABILITY MATRICES AND SIGNATURES}

In this appendix we give some matrix identities among the various stability matrices and the resulting signature properties that are used in Sec. III.B. The properties are a simple consequence of the symplectic structure of canonical transformations. Indeed, let us group the stability matrices into the usual Monodromy matrix

$$
\mathbf{M}=\left(\begin{array}{cc}
\partial_{\mathbf{q}_{0}} \mathbf{q}(t) & \partial_{\mathbf{p}_{0}} \mathbf{q}(t) \\
\partial_{\mathbf{q}_{0}} \mathbf{p}(t) & \partial_{\mathbf{p}_{0}} \mathbf{p}(t)
\end{array}\right) \equiv\left(\begin{array}{cc}
\mathbf{A} & \mathbf{B} \\
\mathbf{C} & \mathbf{D}
\end{array}\right)
$$

M represents a matrix Jacobian of a canonical transformation and hence satisfies the symplectic condition

$$
\mathbf{M}^{T} \mathbf{J M}=\mathbf{J}, \quad \mathbf{M} \mathbf{J} \mathbf{M}^{T}=\mathbf{J},
$$

where

$$
\mathbf{J}=\left(\begin{array}{cc}
\mathbf{0}_{N} & \mathbf{I}_{N} \\
-\mathbf{I}_{N} & \mathbf{0}_{N}
\end{array}\right)
$$

has the well-known [12] properties $\mathbf{J}^{T}=-\mathbf{J}, \mathbf{J}^{2}=-\mathbf{I}_{2 N}$, and $\mathbf{J}^{T} \mathbf{J}=\mathbf{J J}^{T}=\mathbf{I}_{2 N}$. The evolution of $\mathbf{M}$ satisfies the first-order system of differential equations for the stability matrices

$$
\frac{d}{d t} \mathbf{M}=\mathbf{J} \partial_{(\mathbf{q}, \mathbf{p})}^{2} H \mathbf{M}
$$

This equation and its matrix transpose also readily proves Eqs. (C2) since Eq. (C4) gives $d\left(\mathbf{M J} \mathbf{M}^{T}\right) / d t$ $=d\left(\mathbf{M}^{T} \mathbf{J M}\right) / d t=0_{2 N}$ and since $\mathbf{M}\left(t=t_{0}\right)=\mathbf{I}_{2 N}$ so that Eqs. (C2) are obeyed at initial time $t_{0}$ and hence satisfied for all time.

Substituting Eq. (C1) into the first of Eqs. (C2) gives a first set of symmetry relations

$$
\mathbf{A}^{T} \mathbf{C}=\mathbf{C}^{T} \mathbf{A}=\left(\mathbf{A}^{T} \mathbf{C}\right)^{T}, \quad \mathbf{B}^{T} \mathbf{D}=\mathbf{D}^{T} \mathbf{B}=\left(\mathbf{B}^{T} \mathbf{D}\right)^{T}
$$

and substitution into the second of Eqs. (C2) gives

$$
\mathbf{A B} \mathbf{B}^{T}=\mathbf{B} \mathbf{A}^{T}=\left(\mathbf{A} \mathbf{B}^{T}\right)^{T}, \quad \mathbf{C D}^{T}=\mathbf{D C}^{T}=\left(\mathbf{C D}^{T}\right)^{T} .
$$

Multiplying the first relation in Eq. (C5) by $\mathbf{A}^{-1}$ to the right and by $\left(\mathbf{A}^{T}\right)^{-1}$ to the left gives $\mathbf{C A}^{-1}=\left(\mathbf{C A}^{-1}\right)^{T}$. Similar manipulations on the other relations readily show that $\mathbf{D B}{ }^{-1}, \mathbf{A}^{-1} \mathbf{B}$ and $\mathbf{D}^{-1} \mathbf{C}$ are also symmetric matrices, given that the inverses exist. Such symmetry properties are also evident from Eqs. (B3), (B6), (B9), and (B12). The inverses of such matrix multiples are obviously also symmetric since $\left(\mathbf{S}^{-1}\right)^{T}=\left(\mathbf{S}^{T}\right)^{-1}$ for any invertible matrix $\mathbf{S}$.

Equations (C5) and (C6) now lead to the following signature properties. In particular, multiplying the first of Eqs. (C5) on the right by the identity in the form $\mathbf{C}^{-1} \mathbf{C}$ gives $\mathbf{A}^{T} \mathbf{C}=\mathbf{C}^{T}\left(\mathbf{A} \mathbf{C}^{-1}\right) \mathbf{C}$. Then taking signatures while using the properties $\operatorname{sig} \mathbf{S}=\operatorname{sig} \mathbf{P}^{T} \mathbf{S P}$ and $\operatorname{sig} \mathbf{S}=\operatorname{sig} \mathbf{S}^{-1}$, for any invertible matrix $\mathbf{P}$ and symmetric $\mathbf{S}$, shows that

$$
\operatorname{sig} \mathbf{A}^{T} \mathbf{C}=\operatorname{sig} \mathbf{A} \mathbf{C}^{-1}=\operatorname{sig} \mathbf{C A} \mathbf{A}^{-1} .
$$

Similar manipulations on the other relations readily give the rest of the useful symmetry relations,

$$
\begin{aligned}
& \operatorname{sig} \mathbf{B}^{T} \mathbf{D}=\operatorname{sig} \mathbf{B} \mathbf{D}^{-1}=\operatorname{sig} \mathbf{D} \mathbf{B}^{-1}, \\
& \operatorname{sig} \mathbf{A} \mathbf{B}^{T}=\operatorname{sig} \mathbf{B}^{-1} \mathbf{A}=\operatorname{sig} \mathbf{A}^{-1} \mathbf{B},
\end{aligned}
$$

and

$$
\operatorname{sig} \mathbf{C D}^{T}=\operatorname{sig} \mathbf{C}^{-1} \mathbf{D}=\operatorname{sig} \mathbf{D}^{-1} \mathbf{C} .
$$

[1] J. H. Van Vleck, Proc. Natl. Acad. Sci. U.S.A. 14, 178 (1928).

[2] V. P. Maslov, USSR Comput. Math. Math. Phys. 3, 744 (1962).

[3] M. Gutzwiller, Math. Phys. 8, 1979 (1967).

[4] V. P. Maslov and M. V. Fedoryuk, Semiclassical Approximation in Quantum Mechanics (Reidel, Boston, 1981).

[5] G. Campolieti and P. Brumer, Phys. Rev. A 50, 997 (1994).

[6] M. V. Fedoryuk, USSR Comput. Math. Math. Phys. 2, 152 (1963); J. N. L. Connor, Mol. Phys. 25, 181 (1973).
[7] N. Bleistein and R. A. Handelsman, Asymptotic Expansions of Integrals (Dover, New York, 1986); A. Erdélyi, Asymptotic Expansions (Dover, New York, 1956).

[8] Similar identities were employed by L. Hörmander, Acta Math. 127, 79 (1971); D. Fujiwara, Nagoya Math. J. 124, 61(1991).

[9] Coordinates of any trajectory generated by the dynamics for a time $t-t_{0}$ and initiated at any point $\mathbf{q}\left(t_{0}\right)=\mathbf{q}_{0}, \mathbf{p}\left(t_{0}\right)=\mathbf{p}_{0}$ at time $t_{0}$ are denoted explicitly as functions of these variables 
when written as $\mathbf{q}(t)=\mathbf{q}\left(\mathbf{q}_{0}, \mathbf{p}_{0} ; t, t_{0}\right)$ and similarly for the momenta $\mathbf{p}(t)=\mathbf{p}\left(\mathbf{q}_{0}, \mathbf{p}_{0} ; t, t_{0}\right)$.

[10] W. H. Miller, Adv. Chem. Phys. 25, 69 (1974).

[11] L. S. Schulman, Techniques and Applications of Path Integration (Wiley, New York, 1981).

[12] H. Goldstein, Classical Mechanics, 2nd ed. (Addison-Wesley, Reading, MA, 1980); V. I. Arnold, Mathematical Methods in
Classical Mechanics (North-Holland, New York, 1980); E. T. Whittaker, A Treatise on the Analytical Dynamics of Particles and Rigid Bodies, 4th ed. (Cambridge University Press, Cambridge, 1937).

[13] See also S. Levit, K. Möhring, U. Smilansky, and T. Dreyfus, Ann. Phys. (N.Y.) 114, 223 (1978).

[14] See also the recent review of Maslov theory by R. G. Littlejohn, J. Stat. Phys. 68, 7 (1992). 